\title{
Oxygen and magnesium abundance in the ultra-metal-poor giants CS 22949-037 and CS 29498-043: Challenges in models of atmospheres
}

\author{
G. Israelian ${ }^{1}$, N. Shchukina ${ }^{2}$, R. Rebolo ${ }^{1,3}$, G. Basri ${ }^{4}$, J. I. González Hernández ${ }^{1}$, and T. Kajino ${ }^{5}$ \\ 1 Instituto de Astrofísica de Canarias, 38205 La Laguna, Tenerife, Spain \\ 2 Main Astronomical Observatory, National Academy of Sciences, 03680 Kyiv-127, Ukraine \\ ${ }^{3}$ Consejo Superior de Investigaciones Científcas, Spain \\ 4 Astronomy Department, University of California, Berkeley, California 94720, USA \\ 5 National Astronomical Observatory of Japan, 2-21-1, Osawa, Mitaka, Tokyo 181-8588, Japan
}

Received 2 December 2003 / Accepted 23 February 2004

\begin{abstract}
We report the results of a non-LTE Fe, $\mathrm{O}$ and $\mathrm{Mg}$ abundance analysis of the carbon-nitrogen-rich ultra-metal-poor giants CS 29498-043 and CS 22949-037. The abundance of oxygen has been derived from measurements of the oxygen triplet at 7771-5 ̊̊ in high resolution spectra obtained with KeckI/HIRES and the forbidden line [O I] 6300 A detected in the TNG/SARG spectra of CS 29498-043. Detailed non-LTE analysis of Fe lines has provided reliable stellar parameters which, however, do not resolve the oxygen abundance conflict as derived from the O I 7771-5 $\AA$ triplet and the [O I] $6300 \AA$ forbidden lines. We obtained the following oxygen abundance: for CS 22949-037 [O/Fe] = 3.13, 1.95; and for CS 29498-043; [O/Fe] = 3.02, 2.49, based on the O I 7771-5 A triplet and the [O I] 6300 A forbidden line, respectively. A similar conflict appears to exist between the forbidden resonance line $\mathrm{Mg}$ I $4571 \AA$ and several subordinate lines, such as Mg I 5172 and $5183 \AA$. Our analysis demonstrates the failure of standard plane-parallel atmosphere models to describe the physical conditions in the line-forming regions of these ultra-metal-poor giants.
\end{abstract}

Key words. Galaxy: evolution - nuclear reactions, nucleosynthesis, abundances - stars: abundances stars: late-type - stars: population III - stellar atmospheres

\section{Introduction}

Surveys of metal-poor stars (Beers et al. 1992) and their abundance studies (Ryan et al. 1996; Norris et al. 1999) are aimed at investigating the chemical evolution of the Galaxy and the nucleosynthetic yields of supernovae. Despite the numerous studies in this field, the current situation with abundance trends of various $\alpha$-elements is very confusing. Observations of Stephens \& Boesgaard (2002) demonstrate that the $[\alpha / \mathrm{Fe}]$ ratios for $\mathrm{Ca}, \mathrm{Si}, \mathrm{Ti}$ and $\mathrm{Mg}$ do not show a flat plateau at $[\mathrm{Fe} / \mathrm{H}]<-1$, as was claimed in previous studies. The results obtained by Idiart \& Thevenin (1999) and Stephens \& Boesgaard (2002) cannot be called consistent with the analysis presented by Carretta et al. (2002) and many others (see McWilliam 1997). The situation with oxygen is far from being resolved (Israelian et al. 1998; Israelian et al. 2001; Nissen et al. 2002; Takeda 2003; Fulbright \& Johnson 2003), while a new debate over the sulfur abundance in metal-poor stars has already emerged (Israelian \& Rebolo 2001; Takada-Hidai et al. 2002; Nissen et al. 2003). These and many other studies clearly show that $[\alpha / \mathrm{Fe}]>0$ for the great majority of metal-poor stars in the Galaxy. However, it is hard to speak of any trend when

Send offprint requests to: G. Israelian, e-mail: gil@iac.es the abundance ratios in many stars computed by different authors disagree by more than $0.3-0.4$ dex. There are many aspects to this serious problem, and we shall not discuss them further here. Abundance analysis of ultra-metal-poor stars with $[\mathrm{Fe} / \mathrm{H}]<-3$ gives rise to even more enigmas into this field.

It is well known that the chemical composition of the atmospheres of halo dwarfs is not altered by any internal mixing and therefore provides a good opportunity to constrain Galactic chemical evolution models. Unfortunately, most of the known ultra-metal-poor stars are not dwarfs but giants (Beers et al. 1992), which pose two serious problems. First, the atmospheric parameters of giants are more uncertain and second, their surfaces can be polluted by enriched material that has been either dredged from the stellar interior or transferred from a companion star. Oxygen is a key element in this scheme as it can help to distinguish between pristine and pollution origins of other elements and also show which of the aforementioned processes was dominant. There have been intensive investigations of the oxygen abundances in halo stars over the last five years. Abundances derived from near-UV OH lines in metalpoor dwarf stars (Israelian et al. 1998, 2001; Boesgaard et al. 1999) show that the $[\mathrm{O} / \mathrm{Fe}]\left([\mathrm{O} / \mathrm{Fe}]=\log (\mathrm{O} / \mathrm{Fe})_{\star}-\log (\mathrm{O} / \mathrm{Fe})_{\odot}\right)$ 
ratio increases from 0 to 1 between $[\mathrm{Fe} / \mathrm{H}]=0$ and -3 . The abundances derived from low-excitation $\mathrm{OH}$ lines agreed well with those derived from high-excitation lines of the O I triplet at 7771-5 $\AA$ (Israelian et al. 1998, 2001; Boesgaard et al. 1999; Nissen et al. 2002). It seems that even the [OI] forbidden line at $6300 \AA$ supports the "quasi-linear" trend of [O/Fe] (Nissen et al. 2002) when standard 1D atmospheric models are employed. While some authors claim a good agreement between the forbidden line [OI] $6300 \AA$ and the near-IR triplet (Mishenina et al. 2000; Nissen et al. 2002), others suggest the opposite (Carretta et al. 2000). In a recent study Takeda (2003) found that the disagreement between the triplet and the forbidden line tends to be larger for cool giants. Fulbright \& Johnson (2003) support this conclusion in a detailed study of 55 subgiants and giants. These authors conclude that it is impossible to resolve the disagreement in the two indicators without adopting an ad hoc temperature scale that is incompatible with standard temperature scales such as IRFM and $\mathrm{H} \alpha$. In fact, the $[\mathrm{O} / \mathrm{Fe}]$ trend obtained based on the ad hoc scale (see Fig. 13 of Fulbright \& Johnson 2003) is identical to the trends presented by Israelian et al. (2001) and Nissen et al. (2002). There is no plateau at $[\mathrm{O} / \mathrm{Fe}]=0.5$. Despite considerable observational effort the trend of the $[\mathrm{O} / \mathrm{Fe}]$ ratio in the halo is still unclear. However, the latest studies (Israelian et al. 2001; Nissen et al. 2002; Takeda 2003; Fulbright \& Johnson 2003) suggest that dwarfs provide more reliable and consistent abundances than giants. It is not clear how the $3 \mathrm{D}$ effects will resolve this conflict since the latter do not predict an agreement between different oxygen abundance indicators in dwarfs (Asplund \& García Pérez 2002).

McWilliam et al. (1995) were the first to carry out a detailed spectroscopic analysis of CS 22949-037 and to confirm that the star is very metal-poor with an $\alpha$-element excess. Furthermore, Depagne et al. (2002) performed a more detailed investigation of this object and found a large excess of oxygen $([\mathrm{O} / \mathrm{Fe}]=2.0)$ and sodium $([\mathrm{Na} / \mathrm{Fe}]=2.1)$. Zero-heavy-element supernovae models with fall back have been invoked in order to interpret the elemental abundance ratios in this star. Aoki et al. (2002) have presented a detailed analysis of another ultra-metal-poor giant CS 29498-043 with a very high abundance excess of $[\mathrm{Mg} / \mathrm{Fe}]=1.81$. Both, CS 22949-037 and CS 29498-043 exhibit a large overabundance of $\mathrm{N}$ and $\mathrm{C}$ but show no significant enhancement of neutron-capture elements. It is possible that the surfaces of these stars have been polluted by enriched material, either dredged from the star's inner core or transferred from a companion star. The abundances of $\alpha$-elements could be used to discriminate in favour of one of these hypotheses or to confirm a pristine origin. The detailed comparison of elemental abundances may provide important constraints on the properties of the first supernova progenitors.

In this article we present observations of the oxygen triplet in CS 22949-037 and CS 29498-043, as well as the detection of the forbidden line [O I] $6300 \AA$ in CS 29498-043. The stellar parameters and the abundances of iron, oxygen and magnesium were derived in non-LTE. We report a significant discrepancy between the abundances derived from the oxygen triplet and the forbidden line. The conflict cannot be resolved under any circumstances, at least for CS 22949-037. A similar conflict was found for $\mathrm{Mg}$. We question the validity of standard plane-parallel models of atmospheres employed in the present analysis.

\section{Observations}

The observations of CS 29498-043 and CS 22949-037 were performed on 2002 October 31 and 2002 May 20 (only CS 22949-037) at the Keck I using the high-resolution spectrograph HIRES and the TEK $2048 \times 2048$ pixel $^{2}$ CCD. A 1.1 arcsec entrance slit provided a resolving power $R \sim 60000$. A red wavelength setting was used to observe oxygen triplet lines at 7771-5 $\AA$. The average signal-to-noise $(S / N)$ ratio of the combined spectrum near $7770 \AA$ was $S / N>100$. Five spectra of CS 29498-043 with a total exposure time $13500 \mathrm{~s}$ were obtained on 2002 September 26 and 27 with the configuration CD4 at TNG/SARG (La Palma). A resolving power of 29000 was achieved with a 1.6 arcsec slit. The [O I] $6300 \AA$ was not blended with any telluric features in this spectral window and the sky emission at $6300 \AA$ was carefully subtracted using the off-slit spectra. The forbidden line with an equivalent width $E W=60 \pm 10 \mathrm{~m} \AA$ was observed in every exposure, providing an independent confirmation of the detection. Rotational velocity of CS 29498-043 derived from the triplet (HIRES) and the forbidden line (SARG) was $10 \pm 3$ and $8 \pm 3 \mathrm{~km} \mathrm{~s}^{-1}$, respectively. The $S / N$ ratio 30 was reached near $6300 \AA$ in the unbind spectrum of CS 29498-043. However, given the rotational velocity of the star we could apply a factor of two bining providing $S / N \sim 40$ to the final spectrum. All the spectra were reduced using standard IRAF ${ }^{1}$ procedures (bias subtraction, flat-field correction, and extraction of one-dimensional spectra). Different spectra for each object were co-added before wavelength calibration and continuum normalization.

\section{Stellar parameters from the non-LTE computations of iron}

It is well known that a strong over-ionization of neutral iron in metal-poor stars leads to the systematic difference in abundances determined from the Fe I and Fe II lines. This difference increases with decreasing metallicity and may reach 0.4 dex in very metal-poor dwarfs (Thévenin \& Idiart 1999). In addition, the non-LTE modeling predicts a dependence of the non-LTE abundance corrections of Fe I lines on the lower excitation potential $(\chi)$. The corrections are particularly large for the low-excitation Fe I lines, while the non-LTE effects are not important for the Fe II lines. The non-LTE abundance corrections in the Sun are in the range 0.02-0.1 dex (Shchukina \& Trujillo Bueno 2001). In general, non-LTE effects play a significant role in metal-poor stars because of decrease in electron density when collisions with free electrons no longer dominate the kinetic equilibrium. Another consequence of the metal

\footnotetext{
1 IRAF is distributed by the National Optical Astronomical Observatories, which is operated by the Association of Universities for Research in Astronomy, Inc., under contract with the National Science Foundation, USA.
} 
deficiency is an appreciable weakening in UV blanketing. NonLTE over-ionization of Fe I substantially reduces the UV line opacity and allows more flux to escape. These effects lead us to suspect that the gravities of metal-poor giants derived from the LTE Fe analysis are underestimated because of the neglect of non-LTE effects. This effect has been studied in metal-poor dwarfs (Thévenin \& Idiart 1999) and in the subgiant BD +23 1330 (Israelian et al. 2001). In fact, Thévenin \& Idiart (1999) derived gravity corrections of up to 0.5 dex with respect to LTE values, for the case of stars with $[\mathrm{Fe} / \mathrm{H}] \sim-3.0$. They have shown that non-LTE effects are important in determining stellar parameters from the iron ionization balance.

The stellar parameters of our targets were obtained using the ionization equilibrium of $\mathrm{Fe}$. The $\mathrm{Fe}$ model atom used in our study provides very consistent results for a 3D atmospheric model of the Sun (Shchukina \& Trujillo Bueno 2001). The microturbulent velocity was fixed at $2 \mathrm{~km} \mathrm{~s}^{-1}$. NonLTE analysis of Fe based on plane-parallel atmosphere models of Kurucz (1992) was carried out with the code NATAJA (Shchukina \& Trujillo Bueno 2001), and the atmospheric parameters were derived using the same method as in Israelian et al. (2001). The equivalent widths of 26 (CS 29498-043) and 60 (CS 22949-037) Fe lines listed in Tables 1 and 2 were taken from the articles of Aoki et al. (2002) and Depagne et al. (2002), respectively. In the present analysis we used solar abundances from Grevesse \& Sauval (1998). The only exception was oxygen, for which we used the solar abundance $\log \epsilon(\mathrm{O})=8.74$ from Nissen et al. (2002).

Figures 1 to 6 show our computations for a grid of atmospheric models for CS 22949-037 and CS 29498-043. The non-LTE corrections to $[\mathrm{Fe} / \mathrm{H}]$ are around $0.3-0.4$ dex (Figs. 3 and 6) and abundance scatter obtained for the best parameter sets are less than 0.2 dex (Figs. 2 and 5). After many iterations with different input parameters, our final results were $T_{\text {eff }}=4900 \pm 125 \mathrm{~K}, \log g=2.5 \pm 0.3$ and $[\mathrm{Fe} / \mathrm{H}]=-3.5 \pm 0.2$ for CS 22949-037 and $T_{\text {eff }}=4300 \pm 160 \mathrm{~K}, \log g=1.5 \pm 0.35$ and $[\mathrm{Fe} / \mathrm{H}]=-3.5 \pm 0.24$ for CS 29498-043. The errors were computed following McWilliam et al. (1995) and assuming those authors $1-\sigma$ uncertainties of $\sim 0.03$ and $\sim 0.07$ dex for the oscillator strengths of the Fe I and Fe II lines, respectively. We also note that the oscillator strengths of the Fe II lines used in our calculations (Fuhr et al. 1988) are very similar to those compiled by McWilliam et al. (1995). The gravities that we obtained are about 1 dex larger compared with those reported by Aoki et al. (2002) and Depagne et al. (2002).

\section{Oxygen}

We have detected all three lines of the oxygen triplet in CS 22949-037 and CS 29498-043 (Fig. 7) and the [O I] $6300 \AA$ line in CS 29498-043 (Fig. 8). The equivalent width of the forbidden line in CS 29498-043 EW $\sim 35 \mathrm{m \AA}$ measured by Aoki et al. (2003) using SUBARU/HDS is smaller than the value obtained from our TNG/SARG. Our TNG/SARG measurement agrees within 3- $\sigma$. This modest agreement is not surprising since the line detected by Aoki et al. (2003) is strongly blended with a telluric feature and our line was affected by sky emission. The relatively large
Table 1. Equivalent widths of the oxygen and iron lines in CS 22949-037. Data for the oxygen triplet comes from this work while the forbidden line $6300 \AA$ and the Fe lines are taken from Depagne et al. (2002). The wavelengths and the excitation energies of the $\mathrm{Fe}$ lines come from Moore (1959). The oscillator strengths of the Fe II and Fe I were taken from Fuhr et al. (1988). The only exception are Fe I 5049.82, 5232.946 (O'Brian et al. 1991) and 5324.185 A (Holweger et al. 1991).

\begin{tabular}{|c|c|c|c|c|}
\hline Ion & $\lambda(\AA)$ & $\log g f$ & $x$ & $E W$ \\
\hline OI & 7771.960 & 0.324 & 9.11 & 41.0 \\
\hline OI & 7774.180 & 0.174 & 9.11 & 30.0 \\
\hline OI & 7775.400 & -0.046 & 9.11 & 19.0 \\
\hline OI & 6300.230 & -9.759 & 0.00 & 5.0 \\
\hline $\mathrm{Fe} I$ & 3899.709 & -1.531 & 0.09 & 102.2 \\
\hline Fe I & 3920.260 & -1.746 & 0.12 & 95.5 \\
\hline $\mathrm{Fe} I$ & 3922.914 & -1.651 & 0.05 & 102.5 \\
\hline $\mathrm{Fe} I$ & 4005.246 & -0.610 & 1.55 & 61.5 \\
\hline $\mathrm{Fe} I$ & 4045.815 & 0.280 & 1.48 & 99.6 \\
\hline $\mathrm{Fe} I$ & 4063.597 & 0.070 & 1.55 & 89.8 \\
\hline $\mathrm{Fe} I$ & 4071.740 & -0.022 & 1.60 & 84.1 \\
\hline $\mathrm{Fe} I$ & 4076.636 & -0.360 & 3.20 & 6.3 \\
\hline $\mathrm{Fe} I$ & 4132.060 & -0.648 & 1.60 & 58.2 \\
\hline $\mathrm{Fe} I$ & 4143.871 & -0.450 & 1.55 & 66.2 \\
\hline $\mathrm{Fe} \mathrm{I}$ & 4147.673 & -2.104 & 1.48 & 7.7 \\
\hline Fe I & 4181.758 & -0.180 & 2.82 & 10.5 \\
\hline $\mathrm{Fe} I$ & 4187.044 & -0.548 & 2.44 & 18.6 \\
\hline Fe I & 4199.098 & 0.250 & 3.03 & 23.9 \\
\hline $\mathrm{Fe} \mathrm{I}$ & 4202.031 & -0.708 & 1.48 & 60.3 \\
\hline Fe I & 4222.219 & -0.967 & 2.44 & 8.3 \\
\hline $\mathrm{Fe} I$ & 4227.434 & 0.272 & 3.32 & 15.1 \\
\hline $\mathrm{Fe} I$ & 4250.125 & -0.405 & 2.46 & 22.6 \\
\hline $\mathrm{Fe} I$ & 4260.479 & -0.020 & 2.39 & 44.2 \\
\hline $\mathrm{Fe} I$ & 4271.159 & -0.349 & 2.44 & 32.7 \\
\hline $\mathrm{Fe} I$ & 4282.406 & -0.810 & 2.17 & 16.0 \\
\hline $\mathrm{Fe} \mathrm{I}$ & 4325.765 & -0.010 & 1.60 & 91.2 \\
\hline $\mathrm{Fe} I$ & 4404.752 & -0.142 & 1.55 & 85.1 \\
\hline $\mathrm{Fe} \mathrm{I}$ & 4415.125 & -0.615 & 1.60 & 61.8 \\
\hline $\mathrm{Fe} I$ & 4447.722 & -1.342 & 2.21 & 6.0 \\
\hline $\mathrm{Fe} I$ & 4461.654 & -3.210 & 0.09 & 33.0 \\
\hline $\mathrm{Fe} I$ & 4528.619 & -0.822 & 2.17 & 21.3 \\
\hline $\mathrm{Fe} I$ & 4871.323 & -0.410 & 2.85 & 10.7 \\
\hline $\mathrm{Fe} I$ & 4872.144 & -0.600 & 2.87 & 7.8 \\
\hline $\mathrm{Fe} I$ & 4891.496 & -0.140 & 2.84 & 19.8 \\
\hline $\mathrm{Fe} I$ & 4920.509 & 0.060 & 2.82 & 23.5 \\
\hline $\mathrm{Fe} I$ & 4994.133 & -3.080 & 0.91 & 7.5 \\
\hline $\mathrm{Fe} \mathrm{I}$ & 5001.871 & -0.010 & 3.87 & 3.1 \\
\hline $\mathrm{Fe} I$ & 5049.825 & -1.355 & 2.27 & 5.5 \\
\hline $\mathrm{Fe} \mathrm{I}$ & 5051.636 & -2.795 & 0.91 & 11.2 \\
\hline $\mathrm{Fe} \mathrm{I}$ & 5068.774 & -1.230 & 2.93 & 3.9 \\
\hline $\mathrm{Fe} I$ & 5110.414 & -3.760 & 0.00 & 15.9 \\
\hline Fe I & 5123.723 & -3.068 & 1.01 & 4.8 \\
\hline $\mathrm{Fe} I$ & 5127.363 & -3.307 & 0.91 & 3.0 \\
\hline $\mathrm{Fe} I$ & 5166.286 & -4.195 & 0.00 & 7.8 \\
\hline $\mathrm{Fe} I$ & 5171.599 & -1.793 & 1.48 & 19.5 \\
\hline $\mathrm{Fe} \mathrm{I}$ & 5194.943 & -2.090 & 1.55 & 6.9 \\
\hline $\mathrm{Fe} I$ & 5232.946 & -0.057 & 2.93 & 15.6 \\
\hline $\mathrm{Fe} \mathrm{I}$ & 5266.562 & -0.490 & 2.99 & 6.2 \\
\hline $\mathrm{Fe} I$ & 5324.185 & -0.100 & 3.20 & 7.2 \\
\hline $\mathrm{Fe} I$ & 5339.935 & -0.680 & 3.25 & 3.2 \\
\hline $\mathrm{Fe} \mathrm{I}$ & 5371.493 & -1.645 & 0.95 & 62.6 \\
\hline $\mathrm{Fe} I$ & 5383.374 & 0.500 & 4.29 & 2.3 \\
\hline $\mathrm{Fe} I$ & 5397.131 & -1.993 & 0.91 & 46.8 \\
\hline $\mathrm{Fe} I$ & 5405.778 & -1.844 & 0.99 & 44.8 \\
\hline $\mathrm{Fe} I$ & 5429.699 & -1.879 & 0.95 & 48.5 \\
\hline $\mathrm{Fe} I$ & 5434.527 & -2.122 & 1.01 & 31.5 \\
\hline $\mathrm{Fe} I$ & 5446.920 & -1.930 & 0.99 & 43.8 \\
\hline $\mathrm{Fe} I$ & 5506.782 & -2.797 & 0.99 & 9.4 \\
\hline Fe II & 4178.85 & -2.480 & 2.57 & 5.0 \\
\hline Fe II & 4233.16 & -2.000 & 2.57 & 20.2 \\
\hline Fe II & 4416.81 & -2.600 & 2.77 & 4.9 \\
\hline Fe II & 4515.33 & -2.480 & 2.83 & 3.4 \\
\hline Fe II & 4520.22 & -2.600 & 2.79 & 2.8 \\
\hline Fe II & 4555.89 & -2.290 & 2.82 & 7.3 \\
\hline
\end{tabular}


Table 2. Equivalent widths of the oxygen and iron lines in CS 29498-043. Oxygen lines are measured by us while the source of Fe the lines is the paper by Aoki et al. (2002). The wavelengths and the excitation energies of the Fe lines come from Moore (1959). The oscillator strengths of the Fe II and Fe I were taken from Fuhr et al. (1988) except Fe I 5049.825 (O'Brian et al. 1991) and $4957.603 \AA$ (Gigas 1988).

\begin{tabular}{|c|c|c|c|c|}
\hline Ion & $\lambda(\AA)$ & $\log g f$ & $\chi$ & $E W$ \\
\hline O I & 7771.960 & 0.324 & 9.11 & 18.0 \\
\hline O I & 7774.180 & 0.174 & 9.11 & 15.0 \\
\hline O I & 7775.400 & -0.046 & 9.11 & 10.0 \\
\hline O I & 6300.230 & -9.759 & 0.00 & 60. \\
\hline $\mathrm{Fe} I$ & 4005.246 & -0.610 & 1.55 & 87.7 \\
\hline $\mathrm{Fe} I$ & 4459.121 & -1.279 & 2.17 & 33.1 \\
\hline $\mathrm{Fe} I$ & 4461.654 & -3.210 & 0.09 & 83.2 \\
\hline $\mathrm{Fe} I$ & 4489.741 & -3.966 & 0.12 & 43.3 \\
\hline $\mathrm{Fe} \mathrm{I}$ & 4528.619 & -0.822 & 2.17 & 52.1 \\
\hline $\mathrm{Fe} \mathrm{I}$ & 4890.762 & -0.430 & 2.86 & 27.6 \\
\hline $\mathrm{Fe} I$ & 4891.496 & -0.140 & 2.84 & 46.0 \\
\hline $\mathrm{Fe} \mathrm{I}$ & 4918.999 & -0.370 & 2.85 & 35.4 \\
\hline $\mathrm{Fe} I$ & 4920.509 & 0.060 & 2.82 & 56.7 \\
\hline $\mathrm{Fe} I$ & 4939.690 & -3.340 & 0.86 & 36.0 \\
\hline $\mathrm{Fe} I$ & 4957.603 & 0.043 & 2.80 & 67.4 \\
\hline $\mathrm{Fe} I$ & 4994.133 & -3.080 & 0.92 & 27.0 \\
\hline $\mathrm{Fe} \mathrm{I}$ & 5012.071 & -2.642 & 0.86 & 55.8 \\
\hline $\mathrm{Fe} I$ & 5049.825 & -1.355 & 2.28 & 27.5 \\
\hline $\mathrm{Fe} \mathrm{I}$ & 5051.636 & -2.795 & 0.91 & 43.6 \\
\hline $\mathrm{Fe} \mathrm{I}$ & 5083.342 & -2.958 & 0.96 & 36.8 \\
\hline $\mathrm{Fe} I$ & 5110.414 & -3.760 & 0.00 & 65.9 \\
\hline $\mathrm{Fe} I$ & 5123.723 & -3.068 & 1.01 & 26.6 \\
\hline $\mathrm{Fe} I$ & 5127.363 & -3.307 & 0.91 & 21.3 \\
\hline $\mathrm{Fe} \mathrm{I}$ & 5166.286 & -4.195 & 0.00 & 36.5 \\
\hline $\mathrm{Fe} I$ & 5171.599 & -1.793 & 1.48 & 34.4 \\
\hline $\mathrm{Fe} \mathrm{I}$ & 5194.943 & -2.090 & 1.55 & 39.9 \\
\hline Fe II & 4522.634 & -2.030 & 2.83 & 28.0 \\
\hline Fe II & 4583.829 & -2.020 & 2.79 & 25.9 \\
\hline Fe II & 4923.921 & -1.320 & 2.88 & 50.1 \\
\hline Fe II & 5018.434 & -1.220 & 2.88 & 60.3 \\
\hline
\end{tabular}

difference may then be related to uncertainties in the corrections for telluric absorption and/or sky emission. The non-LTE computations of the oxygen atom were carried out using the atomic model with 23 levels of O I and one level of O II described by Carlsson \& Judge (1993). While only 31 boundbound and 23 bound-free radiative transitions were considered in our computations, we note that the consideration of additional levels and transitions does not affect our results (Shchukina 1987; Takeda 2003). Our computations for the Sun and metal-poor dwarfs predict non-LTE corrections that are very close to those reported recently by Takeda (2003) and Nissen et al. (2002) for 1D models.

It is well known that inelastic collisions with hydrogen atoms tend to cancel out the non-LTE effects. The forbidden line is not affected by these collisions since it is formed under LTE. The effect on the triplet may reach $0.1 \mathrm{dex}$ in hot and metal-poor subdwarfs such as LP 815-43 (e.g. Nissen et al. 2002). However, it is often stated that Drawin's formalism (Drawin 1968) gives very uncertain results for hydrogen collision rates (e.g. Belyaev et al. 1999). Obviously, we are not concerned with these problems in our targets since the density of $\mathrm{H}$ atoms (and therefore the collision rates) in the atmosphere of $\mathrm{K}$ giants is about two orders of magnitude lower than in dwarfs. Moreover, inelastic collisions with hydrogen atoms bring the oxygen abundance derived from the 7771-5 $\AA$ triplet close to its LTE value. This will make the "oxygen conflict" even more severe as the discrepancy between the triplet and the forbidden line will be greater. Thus, collisions with $\mathrm{H}$ atoms were not taken into account in our computations. We also note that the effects of triplet-quintet system coupling, CO-binding and $L_{\beta}$ pumping are negligible in the atmospheres of our targets.

Our non-LTE oxygen abundances from the near-IR triplet yield $[\mathrm{O} / \mathrm{Fe}]=3.13 \pm 0.21$ and $[\mathrm{O} / \mathrm{Fe}]=3.02 \pm 0.27$ in CS 22949-037 and CS 29498-043, respectively. The non-LTE corrections $(\Delta \epsilon=\epsilon($ non - LTE $)-\epsilon($ LTE $))$ on the triplet and $6300 \AA$ are listed in Table 5. Assuming our stellar parameters for CS 22949-037 and the equivalent width of the forbidden line from Depagne et al. (2002) we find $[\mathrm{O} / \mathrm{Fe}]=1.95$. The large difference between the abundances derived from the triplet and the forbidden line cannot be explained by the non-LTE abundance corrections for the triplet (Table 4) and/or by a noise/telluric correction for the forbidden line. In fact, one needs [OI] $6300 \AA$ line with an $E W>50 \mathrm{~m} \AA$ to provide $[\mathrm{O} / \mathrm{Fe}]>3$. This is clearly ruled out by observations of Depagne et al. (2002). Even if we assume for CS 22949-037 the stellar parameters from Depagne et al. (2002), the difference between $7771-5$ and $6300 \AA$ is as high as 1.55 dex. The forbidden line measured in the SARG spectra of CS 29498-043 has $E W=60 \pm 10 \mathrm{~m} \AA$, providing $[\mathrm{O} / \mathrm{Fe}]=2.49 \pm 0.13$ (Fig. 8).

It is hard to resolve the conflict between the triplet and the forbidden line by playing with the stellar parameters since the errors in $T_{\text {eff }}$ and $\log g$ are of the order of $150 \mathrm{~K}$ and 0.3 dex, respectively. We have to understand the formation mechanisms of these lines in the atmospheres of our targets. The intensity contribution functions (CFI) (see Gray 1976) of the triplet and the forbidden lines decrease rapidly with atmospheric height (Fig. 9), and one can assume, as a working hypothesis, that these lines are optically thin. In this case their equivalent widths will be proportional to the ratio $K^{\text {line }} / K^{\text {cont }}$, where $K^{\text {line }}$ and $K^{\text {cont }}$ are the absorption coefficients in the line and in the continuum, respectively. Many authors state that the strength of the forbidden line is sensitive to the structure of the upper atmosphere. Our analysis (Fig. 9) shows that this is not the case since the forbidden line has a very small oscillator strength and is therefore formed deep in the atmosphere. The triplet lines are formed at this depth because they are excited from high excitation levels which are populated only in the hot layers of the atmosphere. The absorption coefficient in the line will be very sensitive to $T_{\mathrm{e}}$ since the population of the lower level is proportional to $\exp \left(-\chi_{1} / k T_{\mathrm{e}}\right)$ and therefore $E W_{7771-5} \sim T_{\mathrm{e}}$. The strength of the forbidden line will be controlled by $K^{\text {cont }}$ because $K^{\text {line }} \ll K^{\text {cont }}$. Since $K^{\text {cont }} \sim N_{\mathrm{e}}$ (where $N_{\mathrm{e}}$ is the electron density), $E W_{6300} \sim 1 / N_{\mathrm{e}}$. Thus, the $E W_{6300}$ will decrease (while $E W_{7771-5}$ will increase) with an increasing $T_{\mathrm{e}}$ since $N_{\mathrm{e}} \sim T_{\mathrm{e}}$ in stellar atmospheres. Given the $E W \mathrm{~s}$ of the triplet and the forbidden line, one may find the effective 

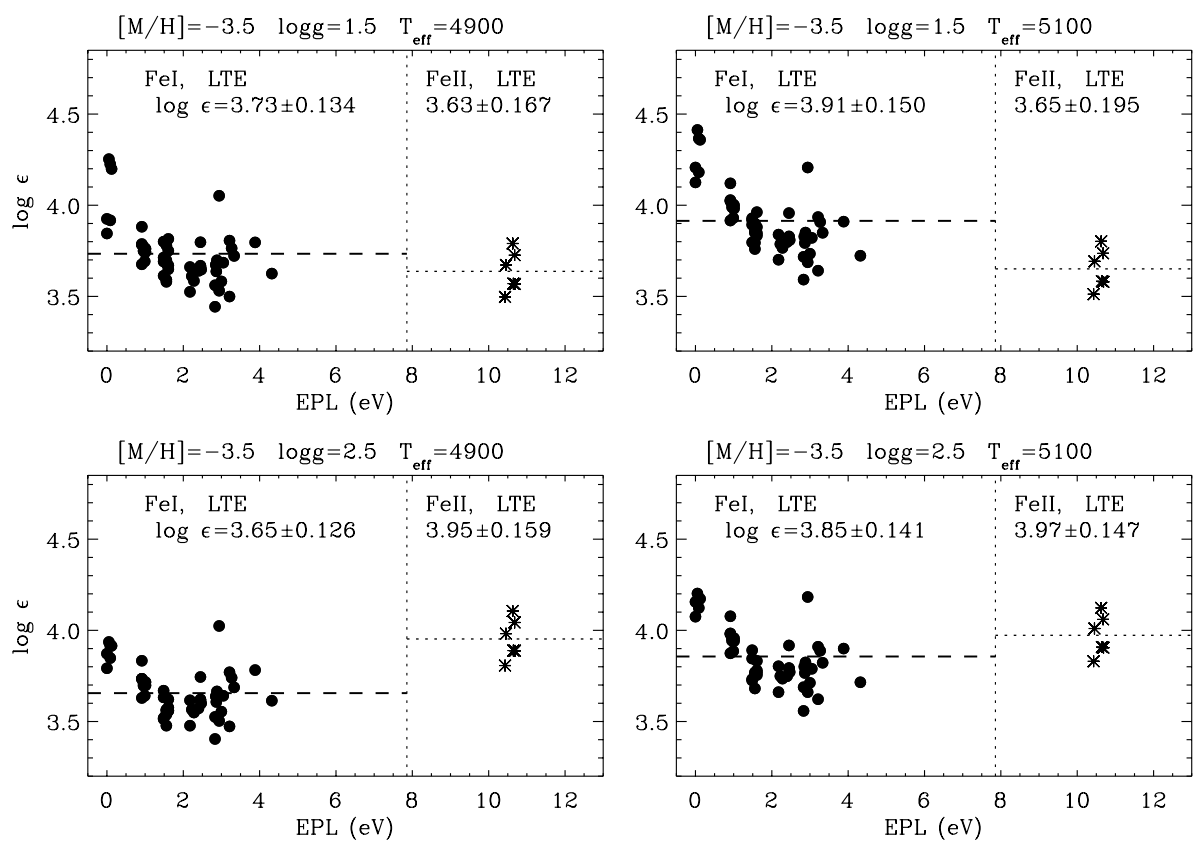

Fig. 1. Results of LTE Fe abundance determination for the Fe I and Fe II lines in CS 22949-037 for a small grid of model atmospheres.
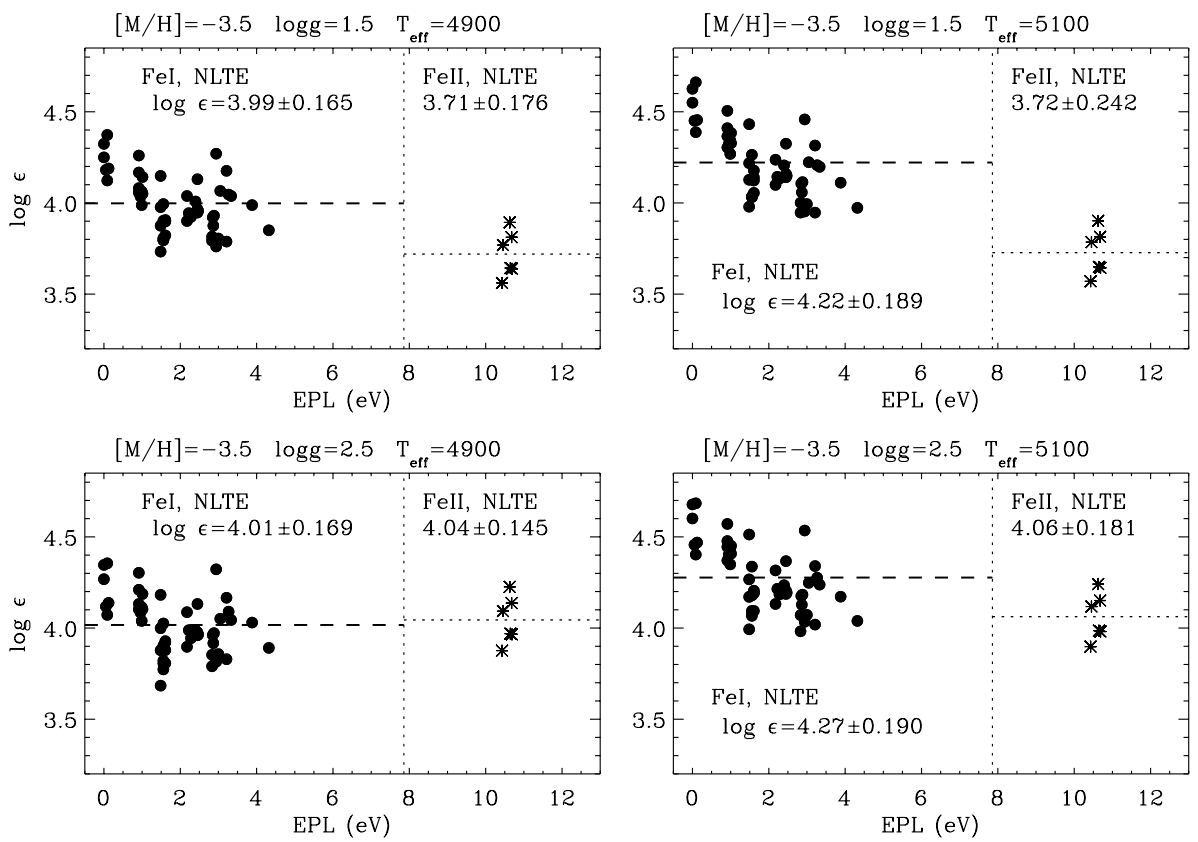

Fig. 2. The same as in Fig. 1 but for a non-LTE Fe analysis.

temperature of the model where these two indicators provide the same abundance. This test has been carried out for CS 22949-037 and the results are shown in Fig. 10. While the $T_{\text {eff }}$ of the star derived from the Fe lines is $4900 \mathrm{~K}$, consistent oxygen abundance can only be obtained when $T_{\text {eff }}=$ $5600 \mathrm{~K}$.

The temperature distributions in two stars with $T_{\text {eff }}=4900$ and $5600 \mathrm{~K}$ and the location of the maximum CFI in the line centre for each model are shown in the Figs. 11 and 12. These figures clearly demonstrate that the formation depth of the forbidden line moves toward the surface and to much higher temperatures in the model with a higher $T_{\text {eff. }}$ In fact, the maximum contribution to the line strength comes from the hot layers
(Fig. 11). The same plot for the near-IR triplet shows (Fig. 12) that the maximum contribution to the equivalent width comes from the layers that are closer to the surface compared with the forbidden line. In fact, our analysis demonstrates that the formation layer of the forbidden line is optically thinner and therefore less sensitive to the temperature gradient (i.e. $\left.T_{\text {eff }}\right)$. Our analysis shows that the failure of the Kurucz (1992) models to provide consistent abundances from the near-IR triplet and the forbidden line, comes from the temperature and density gradients near the continuum-forming region. This problem has the same roots as that related to the discrepancy between the temperatures obtained from colours (continuum) and the Fe ionization balance. It is clear that we cannot resolve the 

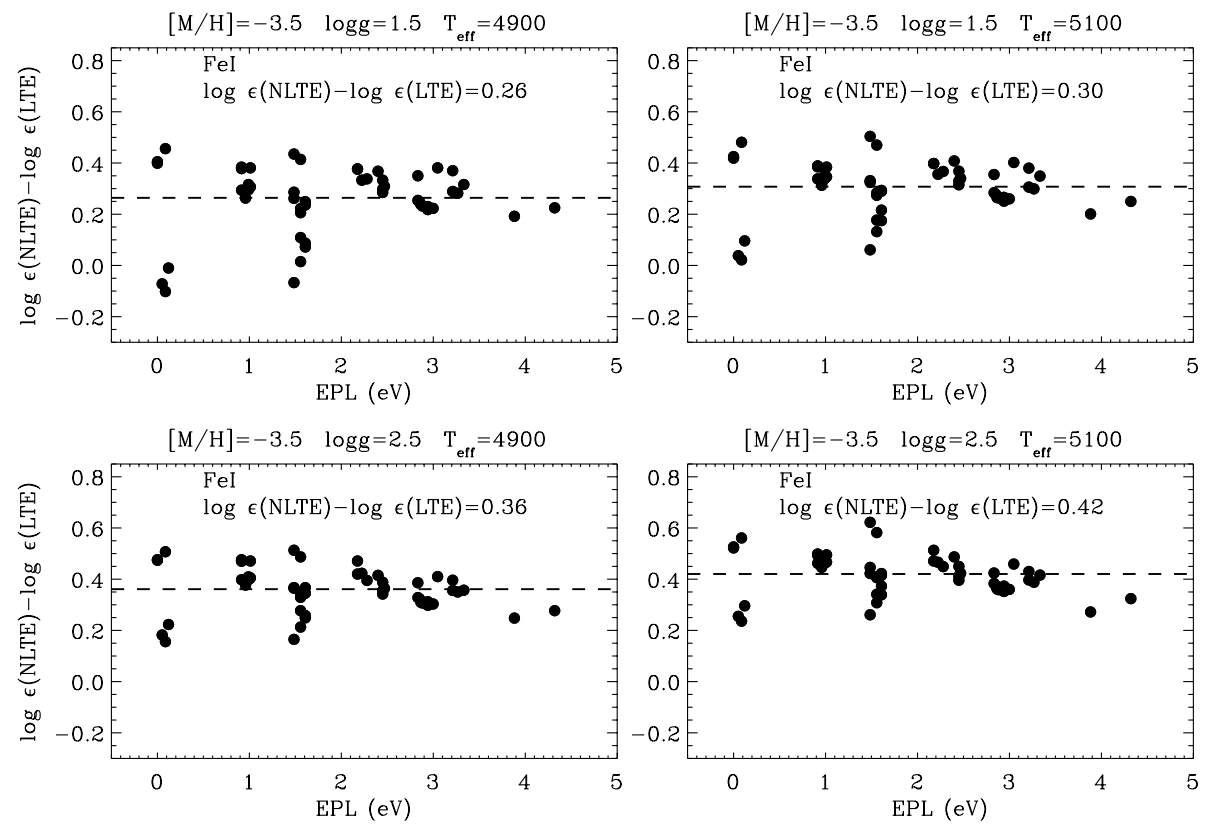

Fig. 3. Difference between non-LTE and LTE abundances as a function of $\chi$ in CS 22949-037.
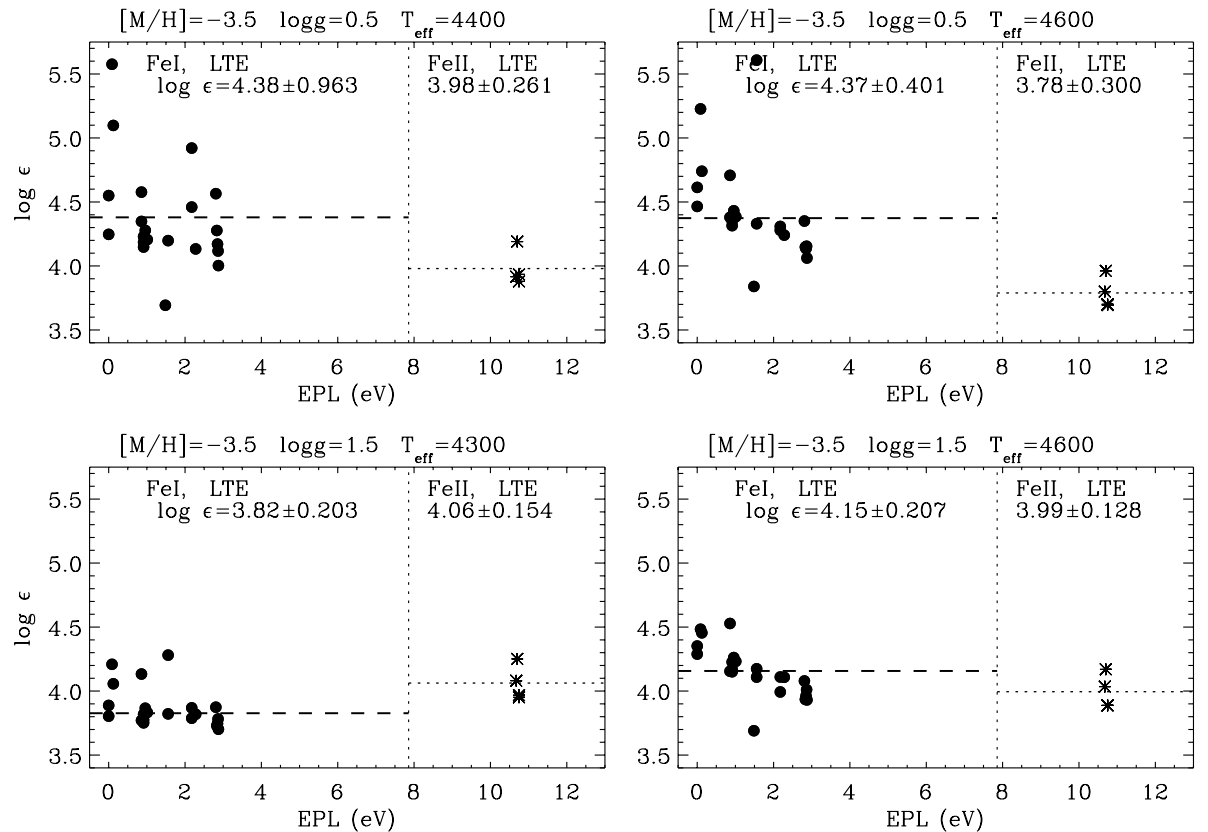

Fig. 4. Results of LTE Fe abundance determination for Fe I and Fe II lines in CS 29498-043 for a small grid of model atmospheres.

conflict between the triplet and the forbidden line by modifying the temperature distribution in the upper atmosphere. The key to this problem is hidden deep in the atmosphere. There must be a certain combination of the electron density and the temperature gradients close to the continuum formation region to provide consistent abundances from the triplet and the forbidden line. It is easy to show that the forbidden line is formed deep in the atmosphere of our targets. We have repeated calculations of this line for CS 22949-037 by gradually removing the upper parts of the atmosphere until we reached those layers where the $6300 \AA$ line is actually "formed". These simple tests have demonstrated that more than $80 \%$ of the $E W$ of the forbidden line is formed at $\log$ mass $>1.4$ (e.g. Fig. 9). The discrepancy between the near-IR triplet and the $6300 \AA$ line also exists in moderately metal-poor giants. These problems have been addressed in detail by Takeda (2003) and Fulbright \& Johnson (2003). Our targets provide the most extreme cases of the "oxygen conflict" at very low metallicities.

We have found that the discrepancy between the near-IR triplet and the forbidden line cannot be explained by non-LTE effects, uncertainties in the stellar parameters or the quality of observations. The oxygen puzzle arises from the failure of standard plane-parallel atmosphere to describe physical conditions in the atmospheres of very metal-poor giants. Spherical effects cannot be the cause of this discrepancy since these lines are formed in very deep and narrow layers where the plane-parallel 

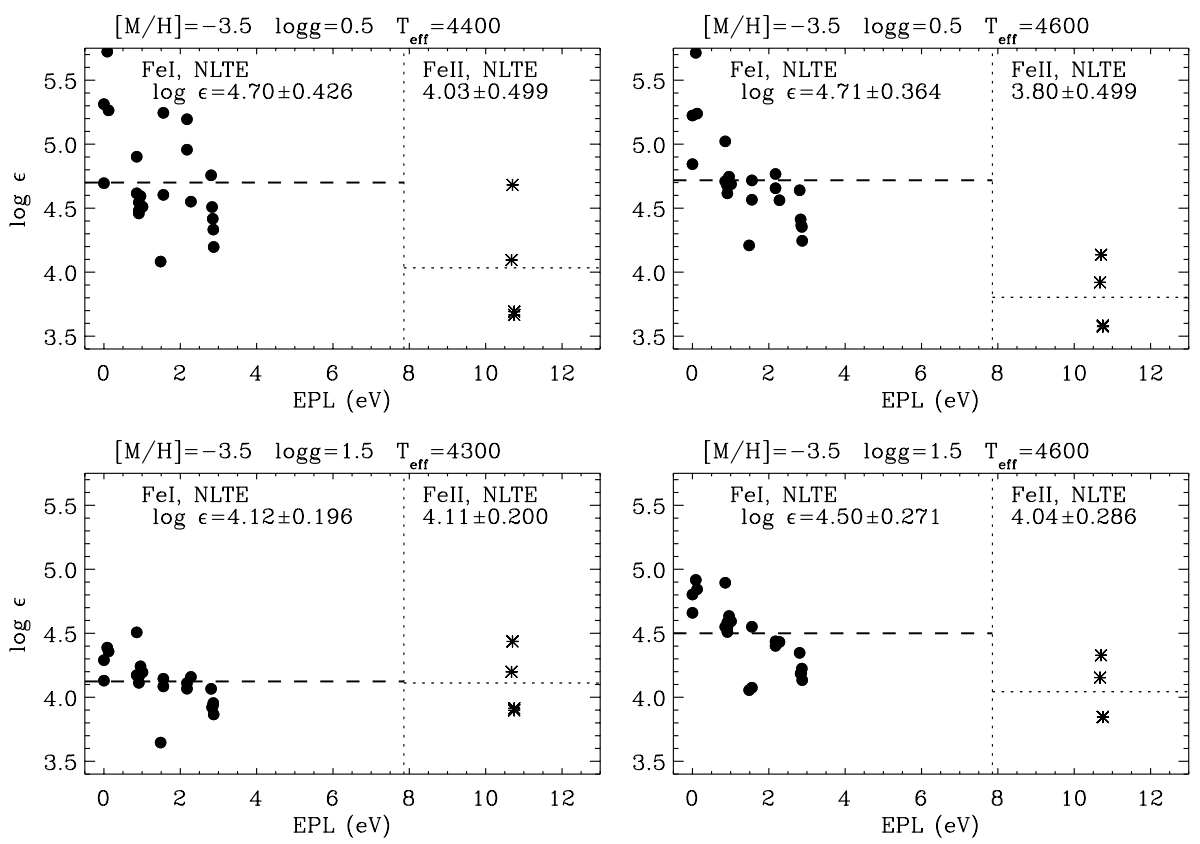

Fig. 5. The same as in Fig. 4 but for a non-LTE Fe analysis.
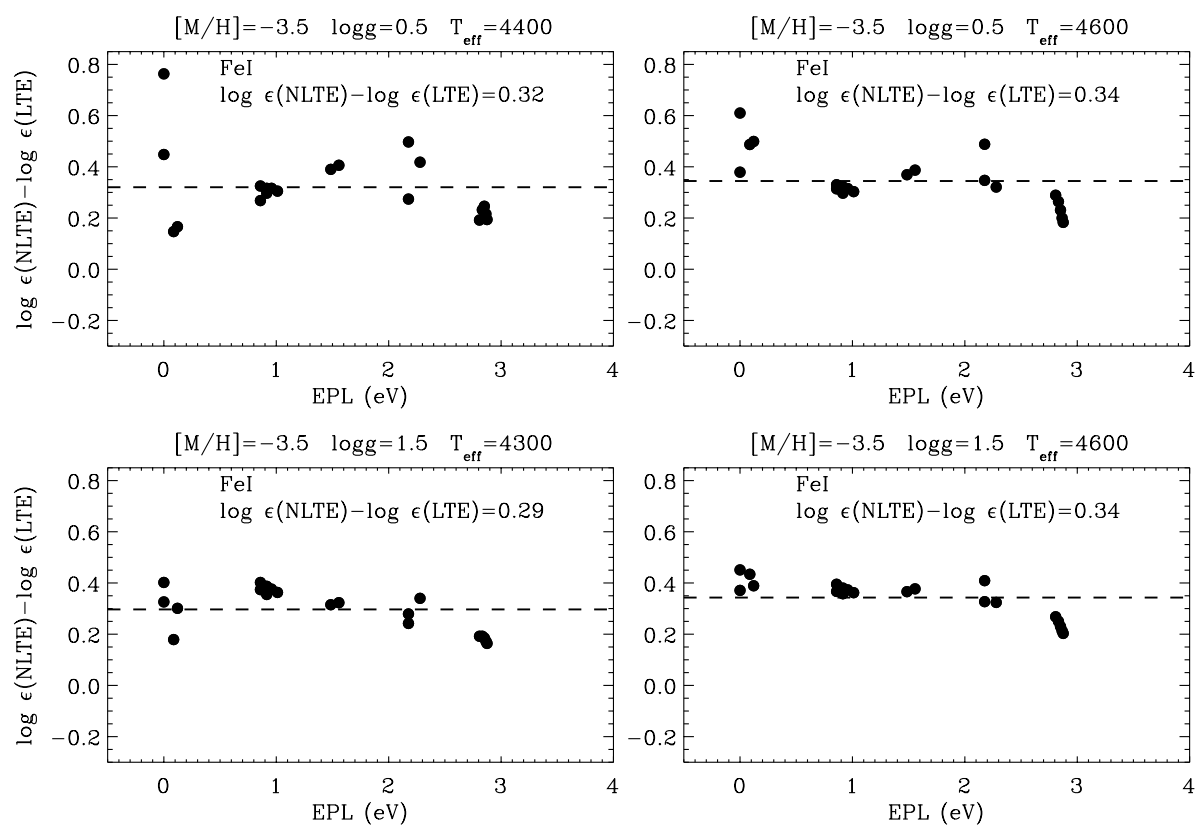

Fig. 6. Difference between non-LTE and LTE abundances as a function of $\chi$ in CS 29498-043.

approximation is certainly applicable. The oxygen puzzle is not unique to CS 22949-037 and CS 29498-043. On a smaller scale it exists in other halo giants (Takeda 2003; Fulbright \& Johnson 2003). We suspect that a similar discrepancy exists for the most metal-poor star in our Galaxy, HE 0107-5240.

\section{Magnesium}

The non-LTE computations of the magnesium atom were carried out using the 24 levels simplified version of the model atom described by Carlsson et al. (1992). Addition of new levels and transitions does not influence on the solution for the lines considered in this paper. The abundance of $\mathrm{Mg}$ in CS 29498-043 was derived from the spectral lines at 4571,5172 and $5183 \AA$ using the equivalent width measurements of Aoki et al. (2002). These lines provide very different abundances just as in the case of oxygen (Table 3 ). The forbidden resonance line $\mathrm{Mg}$ I $4571 \AA$ in CS 29498-043 provides $[\mathrm{Mg} / \mathrm{Fe}]=1.626$ with a non-LTE correction of $\sim 0.18 \mathrm{dex}$ while $\mathrm{Mg}$ I lines at 5172 and $5183 \AA$ provided consistent abundance with a mean $[\mathrm{Mg} / \mathrm{Fe}]=1.08$. The non-LTE corrections in $4571 \AA$ are 0.18 dex while in two other lines they even do not reach -0.1 dex (Table 5). The difference between the 4571 and $5172+5183 \AA$ is larger in the non-LTE than in the LTE case. The mean abundance from the three lines is $[\mathrm{Mg} / \mathrm{Fe}]=1.26$ 


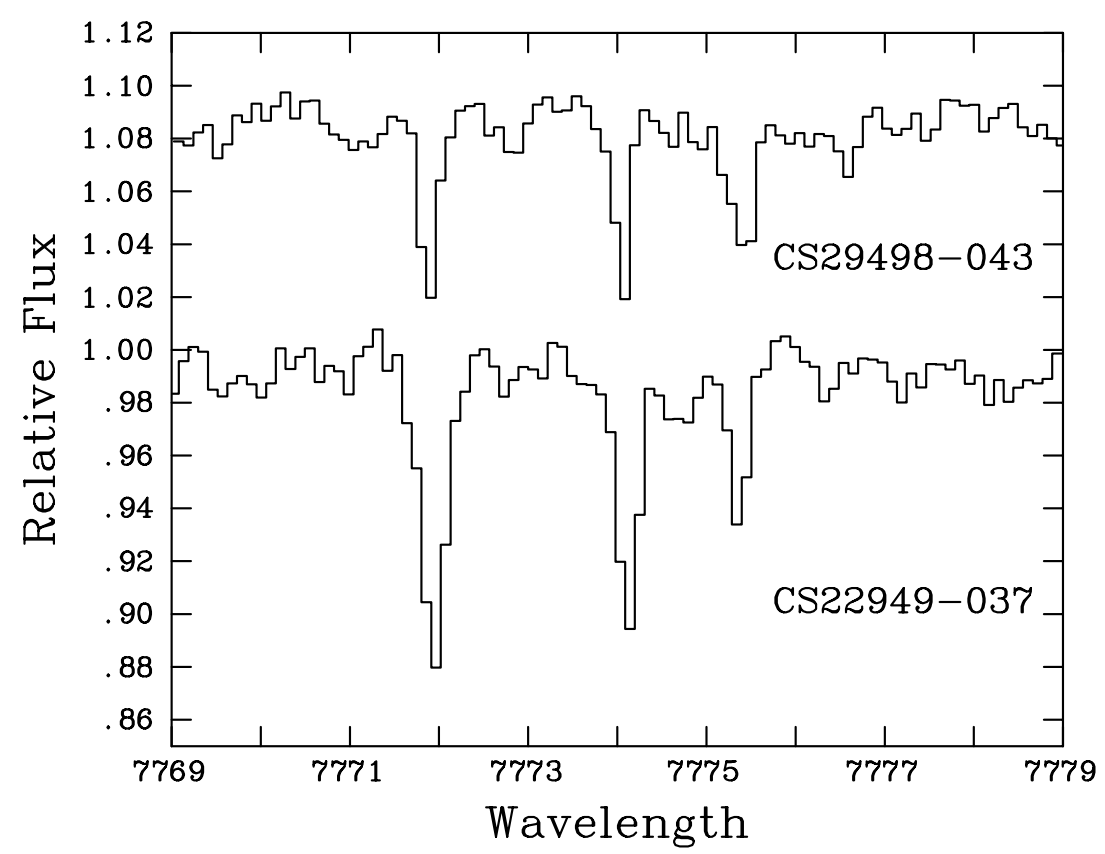

Fig. 7. The oxygen triplet observed in CS 29498-043 (shifted upwards by 0.08) and CS 22949-037.

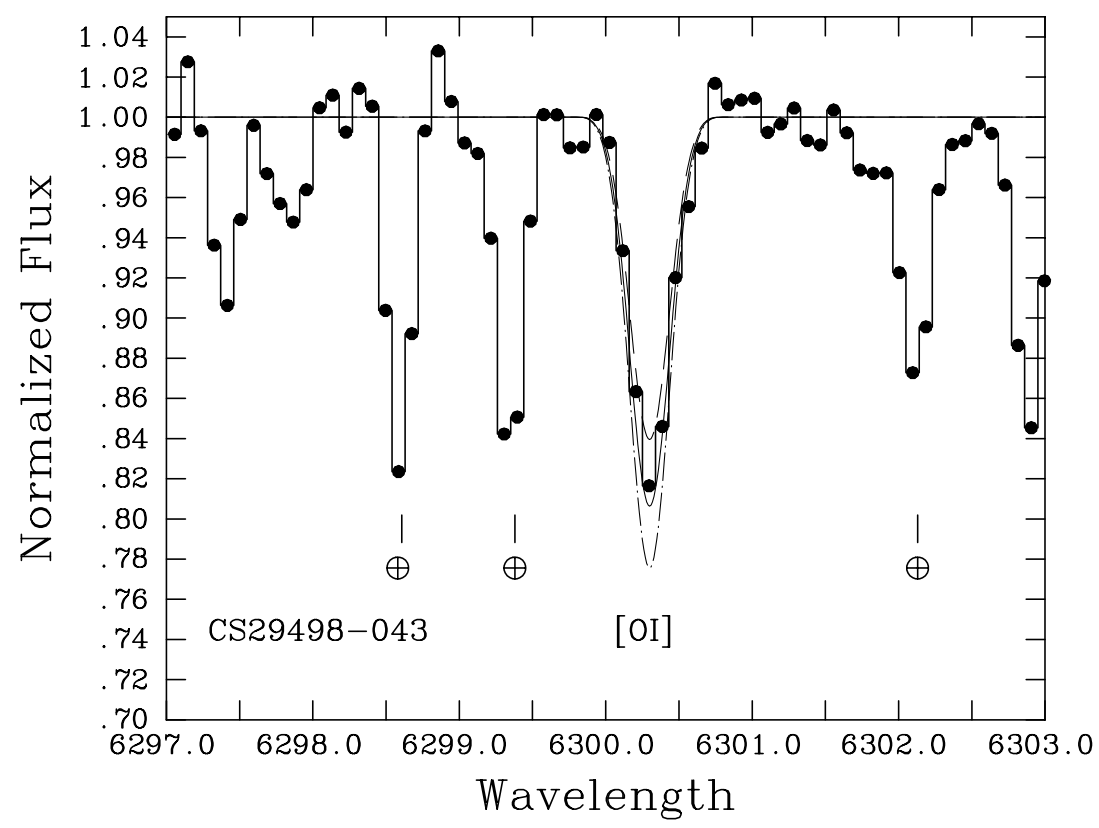

Fig. 8. The oxygen line at $6300 \AA$ A observed in CS 29498-043. Spectrum synthesis was carried out for the values $[\mathrm{O} / \mathrm{H}]=-1.1$ (dashed); -1.0 (solid); -0.9 (dashed-dotted) and assuming $v \sin i=8 \mathrm{~km} \mathrm{~s}^{-1}, V_{\text {macro }}=2 \mathrm{~km} \mathrm{~s}^{-1}$ and 0.6 for the limb-darkening coefficient.

which, of course, does not makes any sense given the huge discrepancy between the 4571 and $5172+5183 \AA$ lines. As for the CS 22949-037, there are six Mg I lines available (Table 4) in our model atom from the article of Depagne et al. (2002). From the five lines listed in Depagne et al. (2002) we obtained a mean $[\mathrm{Mg} / \mathrm{Fe}]=1.156$ while the $4571 \AA$ line again yields a much larger abundance $[\mathrm{Mg} / \mathrm{Fe}]=1.81$.

The CFIs of the 4571 and $5183 \AA$ lines in CS 22949-037 have very different shapes (Fig. 9). The $5183 \AA$ line is formed in an extended region of the upper atmosphere while the forbidden resonance line is produced by the same deep layers where the $6300 \AA$ forbidden line of the neutral oxygen is formed. The $5183 \AA$ line is very strong and less sensitive to the effective temperature compared with the $4571 \AA$ line. The agreement between abundances provided by $4571 \AA$ and $5183 \AA$ lines can be achieved if we increase the temperature in the inner layers of the atmosphere. This will increase the strength of the $4571 \AA$ Aline (and the abundance obtained from this line will 


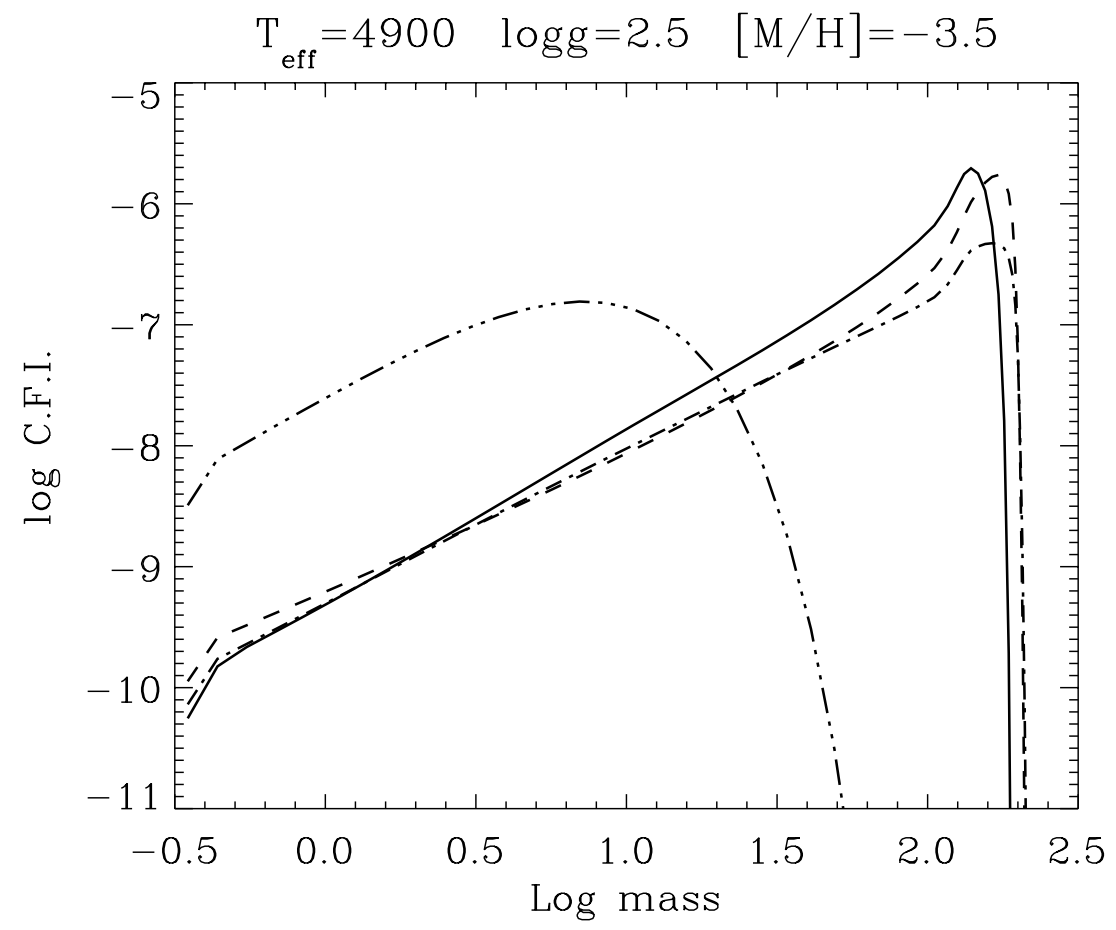

Fig. 9. The intensity contribution functions (CFI) for the line centre in the atmosphere of CS 22949-037 computed for the forbidden line [O I] $6300 \AA$ (dashed line), the near-IR triplet (solid line), the Mg I lines $4571 \AA$ (dashed-dotted) and $5183 \AA$ (dashed-three dotted). The $\log$ mass is above $1 \mathrm{~cm}^{2}$.

Table 3. Non-LTE abundance of oxygen and magnesium lines in CS 29498-043 derived for three sets of the atmospheric parameters. Each set is the best model obtained for a given $[\mathrm{Mg} / \mathrm{Fe}]$ ratio provided on the second line. The second column gives the $E W \mathrm{~s}$ of the $\mathrm{Mg}$ and O lines from Aoki et al. (2002) and this work, respectively.

\begin{tabular}{lcccc}
\hline \hline Line & $\begin{array}{c}E W \\
\mathrm{m \AA}\end{array}$ & $(4400 / 0.5 /-3.5)$ & $(4400 / 1.5 /-3.5)$ & $(4300 / 1.5 /-3.5)$ \\
{$[\mathrm{Mg} / \mathrm{Fe}]=1.8$} & {$[\mathrm{Mg} / \mathrm{Fe}]=1.0$} & {$[\mathrm{Mg} / \mathrm{Fe}]=0$} \\
\hline Mg I 4571 & 104 & 7.286 & 6.233 & 5.626 \\
Mg I 5172 & 218 & 5.031 & 4.905 & 5.147 \\
Mg I 5183 & 228 & 4.937 & 4.785 & 5.012 \\
\hline O I 6300 & 60 & 7.626 & 7.732 & 7.694 \\
O I 7772 & 18 & 7.984 & 8.232 & 8.296 \\
O I 7774 & 15 & 8.019 & 8.274 & 8.343 \\
O I 7775 & 10 & 8.004 & 8.272 & 8.345 \\
\hline
\end{tabular}

decrease) while the $5183 \AA$ will remain almost unchanged. Given the negligible sensitivity of the $5183 \AA$ Aline to the effective temperature one may suggest that this line provides a more reliable abundance. This is similar to the oxygen atom, where the forbidden line is believed to be the best abundance indicator since it is less sensitive to $T_{\text {eff }}$.

$\mathrm{Mg}$ is an important source of free electrons and its overabundance may have a non-negligible effect on the stellar surface gravity derived from the ionization balance of Fe. According Aoki et al. (2002), the effect of Mg overabundance on the gravity of CS 29498-043 is 0.4 dex. We have repeated the non-LTE Fe and Mg analysis of CS 29498-043 for the cases when $[\mathrm{Mg} / \mathrm{Fe}]=1.0$ and $[\mathrm{Mg} / \mathrm{Fe}]=1.8$ (see Figs. 13 and 14).
Table 4. Non-LTE abundance of oxygen and magnesium in CS 22949-037 derived from the model with $T_{\text {eff }}=4900 \mathrm{~K}, \log g=2.5$ and $[\mathrm{Fe} / \mathrm{H}]=-3.5$. The last column gives the abundance correction due to non-LTE effects. The observed $E W \mathrm{~s}$ of Mg and [O I] $6300 \AA$ were taken from Depagne et al. (2002).

\begin{tabular}{lccc}
\hline \hline Line & $E W(\mathrm{~m} \AA)$ & $\log \epsilon$ & $\Delta \epsilon($ NLTE - LTE $)$ \\
\hline Mg I 3829 & 156.1 & 5.224 & 0.187 \\
Mg I 3832 & 185.2 & 4.986 & 0.158 \\
Mg I 3838 & 202.7 & 4.874 & 0.160 \\
Mg I 4571 & 52.9 & 5.810 & 0.670 \\
Mg I 5172 & 176.1 & 5.347 & 0.141 \\
Mg I 5183 & 199.4 & 5.353 & 0.124 \\
\hline O I 6300 & 5. & 7.184 & -0.013 \\
O I 7772 & 41. & 8.430 & -0.238 \\
O I 7774 & 30. & 8.362 & -0.222 \\
O I 7775 & 19. & 8.303 & -0.200 \\
\hline
\end{tabular}

It appears that the gravity of the star is not changed when the $\mathrm{Mg}$ abundance is increased by a factor of 10 (i.e. from $[\mathrm{Mg} / \mathrm{Fe}]=0$ to $[\mathrm{Mg} / \mathrm{Fe}]=1)$. However, the gravity drops to $\log g=0.5$ if we set $[\mathrm{Mg} / \mathrm{Fe}]=1.8$. Thus, the effect is indeed very large if $\mathrm{Mg}$ is as abundant as oxygen. However, as we have already stated, the Mg abundance derived from the $4571 \AA$ line $([\mathrm{Mg} / \mathrm{Fe}]=1.8)$ is most probably overestimated and therefore we do not find it necessary to revise the stellar parameters obtained for $[\mathrm{Mg} / \mathrm{Fe}]=0$ or $[\mathrm{Mg} / \mathrm{Fe}]=1$. The final parameters for CS 29498-043 are assumed $T_{\mathrm{eff}}=4400 \mathrm{~K}, \log g=1.5$ and $[\mathrm{Fe} / \mathrm{H}]=-3.5$ when $[\mathrm{Mg} / \mathrm{Fe}]=1.0$. 


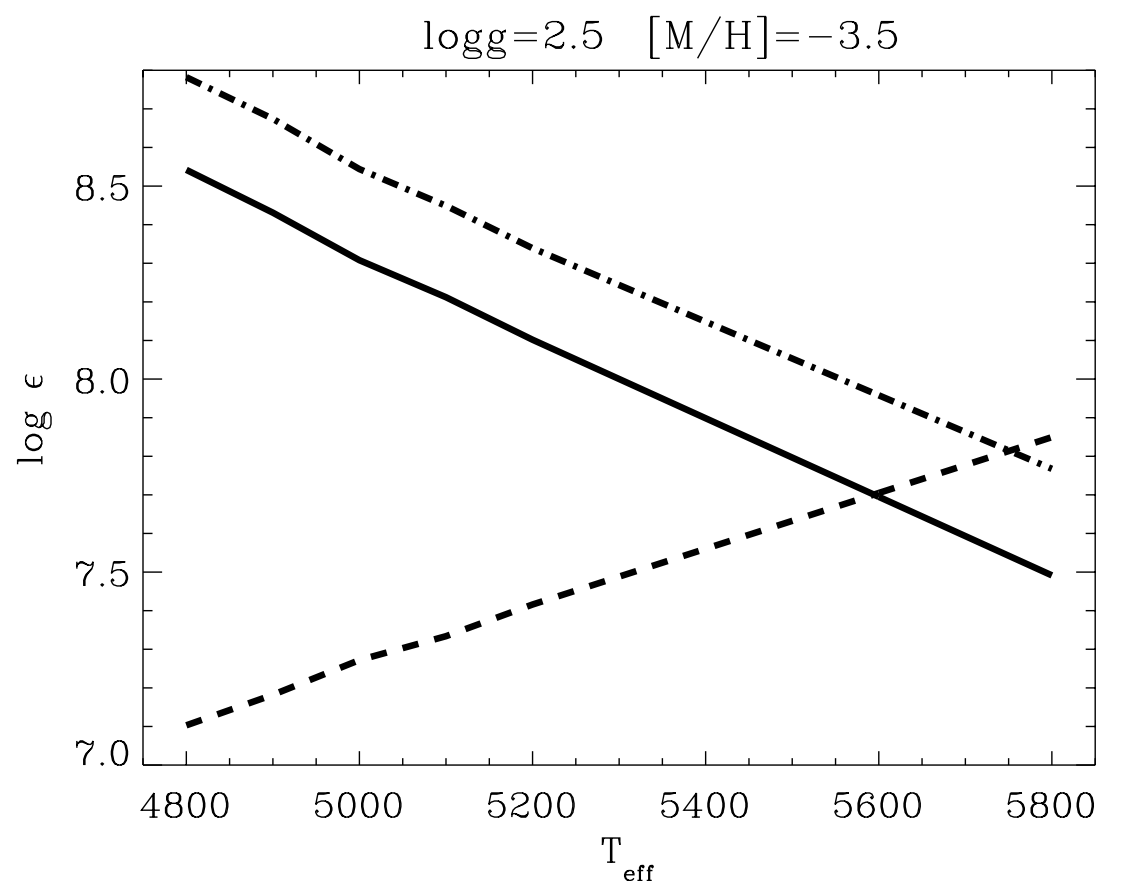

Fig. 10. The oxygen abundance derived from the triplet (non-LTE is a solid line and LTE is dashed-dotted) and the forbidden line (dashed) in CS 22949-037 as function of $T_{\text {eff }}$. The $E W$ s were taken from Table 1.

Table 5. Non-LTE abundance corrections to the oxygen and magnesium lines. $\Delta_{\mathrm{Mg}(\mathrm{Sum})}$ for all $\mathrm{Mg}$ lines except $4571 \AA$ A listed in Tables 3 and 4 . The last two columns provide the abundance differences derived from the triplet and the forbidden line $\left(\triangle A_{\mathrm{O}}\right)$ and from the $4571 \AA$ and the remaining $\mathrm{Mg}$ I lines from Tables 3 and 4.

\begin{tabular}{lcccccc}
\hline \hline Target & $\Delta_{[\mathrm{OI}]}$ & $\Delta_{\text {Triplet }}$ & $\Delta_{\mathrm{Mg} 4571}$ & $\Delta_{\mathrm{Mg}(\mathrm{Sum})}$ & $\Delta A_{\mathrm{O}}$ & $\Delta A_{\mathrm{Mg}}$ \\
\hline CS 29498-043 & -0.012 & -0.188 & 0.179 & -0.071 & 0.53 & 0.546 \\
CS 22949-037 & -0.013 & -0.22 & 0.670 & 0.154 & 1.18 & 0.653 \\
\hline
\end{tabular}

\section{Discussion}

Our results may have an interesting impact on the physics of the supernova progenitor which gave birth to these ultra-metalpoor stars. Should we use the abundance from the triplet or the forbidden line to set constrains on the supernova models? The situation with our targets clearly demonstrates that nonLTE effects on the near-IR oxygen triplet are not responsible for this conflict, and that therefore this abundance indicator is as reliable (or unreliable) as the forbidden line.

Both CS 22949-037 and CS 29498-043 are distinguished from other carbon-rich metal-poor stars with large excesses of neutron capture $s$-process elements. The overabundance of $s$ elements is usually attributed to nucleosynthesis in the thermally pulsing AGB stars. These ultra-metal-poor giants have moderately high abundances of $\mathrm{Mg}, \mathrm{Si}$ and $\mathrm{Al}$, while $\mathrm{C}$ and $\mathrm{N}$ are unusually overabundant. In this paper we find that both stars are also rich in oxygen. It appears that the class of objects with high [O/Fe] is not limited by CS 22949-037 and CS 29498-043. Aoki et al. (2002) proposed the existence of a new class of very metal-poor stars which originate from supernova in which most of the matter were absorbed by the iron core. The most metal-poor star in the Galaxy, HE 0107-5240, also belongs to this class of objects and has a large $[\mathrm{O} / \mathrm{Fe}]=2.4$ derived from the UV OH lines (Bessell et al. 2004). The abundance pattern in this star is consistent with a model (Umeda \& Nomoto 2003) in which the supernova undergoes some mixing followed by a fallback into a massive black hole. However, the oxygen abundance of HE 0107-5240 is smaller than the prediction of Umeda \& Nomoto (2003) by as much as 1 dex. The prototype of this type of supernova is SN 1997D, which was very underluminous because of the small amount $\left(2 \times 10^{-3} M_{\odot}\right)$ of ${ }^{56} \mathrm{Ni}$ ejected during explosion. Four new faint supernova have been reported recently (Pastorello et al. 2003). While such supernovae are not observed frequently, their real number is expected to be much higher because of the faintness of the supernovae. These faint supernovae are expected to be more frequent in the early Galaxy (Umeda \& Nomoto 2003), while their ejecta are characterized by very high $[\mathrm{O} / \mathrm{Fe}]$ ratios. In fact, the monotonically rising trend of $[\mathrm{O} / \mathrm{Fe}]$ can possibly be explained if we assume that the iron yield decreases with stellar mass (i.e. most massive stars form massive black holes). It is clear that the formation rate of massive black holes in the early Galaxy may affect the observed $[\mathrm{O} / \mathrm{Fe}]$ trend. A massive black hole in the low mass X-ray binary system Nova Sco 1994 with $[\mathrm{O} / \mathrm{Fe}]=1.0$ (Israelian et al. 1999) may serve as a prototype for such "failed" supernovae, where almost all the Fe has been accreted by the black hole. Apparently CS 22949-037 is not an "exceptional" star as noted by Depagne et al. (2002). There are another four high [O/Fe] stars: CS 29498-043, HE 0107-5240, CS $29497-$ 030 (Sivarani et al. 2003) and LP 625-44 (Aoki et al. 2002b). Abundances in stars such as LP 625-44 and CS 29497-030 are assumed to result from mass transfer from an AGB star 


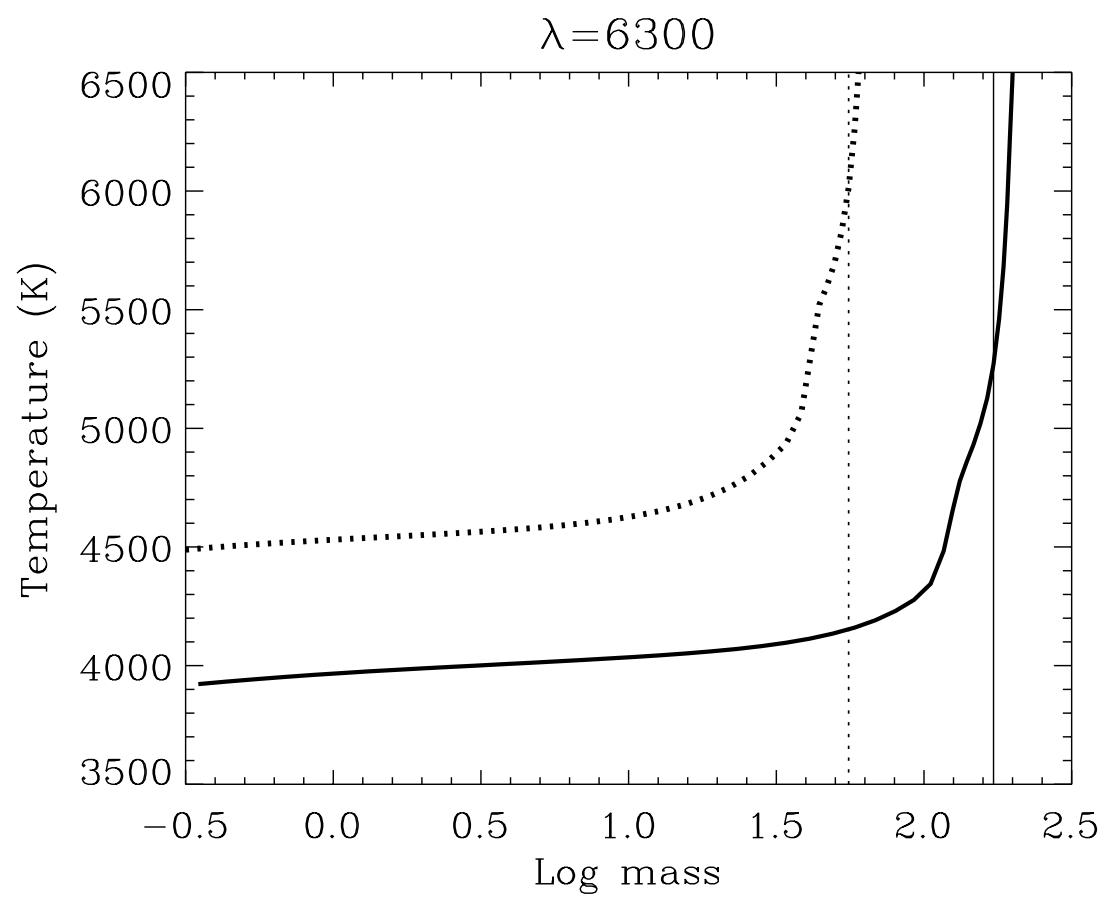

Fig. 11. The temperature distribution for two models with $T_{\text {eff }}=5600 \mathrm{~K}$ (dotted line) and $T_{\text {eff }}=4900 \mathrm{~K}$ (solid line). The dotted and solid vertical lines show the location of the maximum CFI for the forbidden line [O I] $6300 \AA$. The gravity and the metallicity are set at log $g=2.5$ and $[\mathrm{Fe} / \mathrm{H}]=-3.5$, respectively. See Sect. 4 for details. The log mass is above $1 \mathrm{~cm}^{2}$.

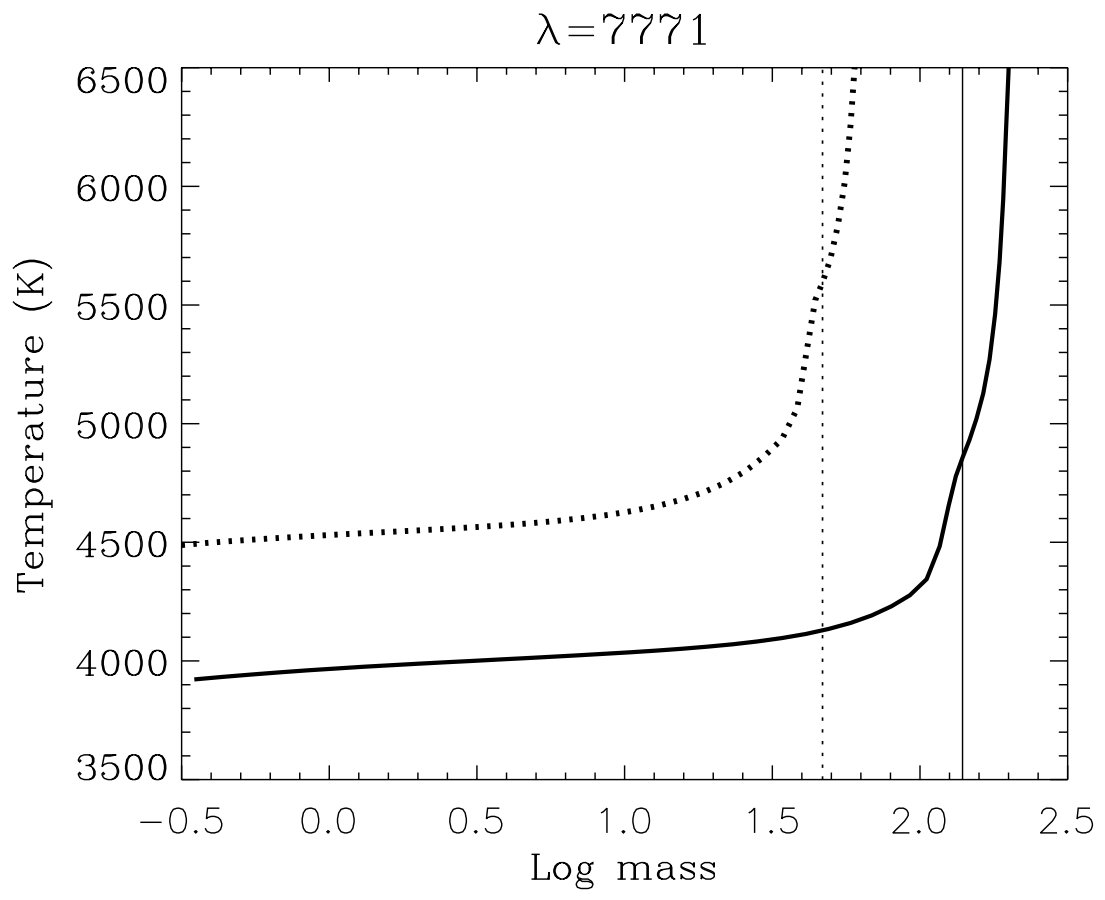

Fig. 12. The same as in Fig. 11 but for the near-IR triplet. The log mass is above $1 \mathrm{~cm}^{2}$.

across a binary on to the observed companion star. However, it is not clear whether this explains the $[\mathrm{O} / \mathrm{Fe}]$ excess in these $s$-process-enhanced stars. Other $s$-process-rich stars such as LP706-7 (Norris et al. 1997) do not show any radial velocity variations. There is much work to be done before we will be able to understand why some $s$-process stars are also oxygen rich. Displaying these stars on the $[\mathrm{O} / \mathrm{Fe}]$ versus $[\mathrm{Fe} / \mathrm{H}]$ diagram with a representative sample of halo dwarfs and giants from the literature (e.g. Nissen et al. 2002; Cayrel et al. 2003; Israelian et al. 2001), we can see the general trend of oxygen in the galaxy and the relative position of these "extreme" stars with respect measurements of other "normal" stars (Fig. 15). 

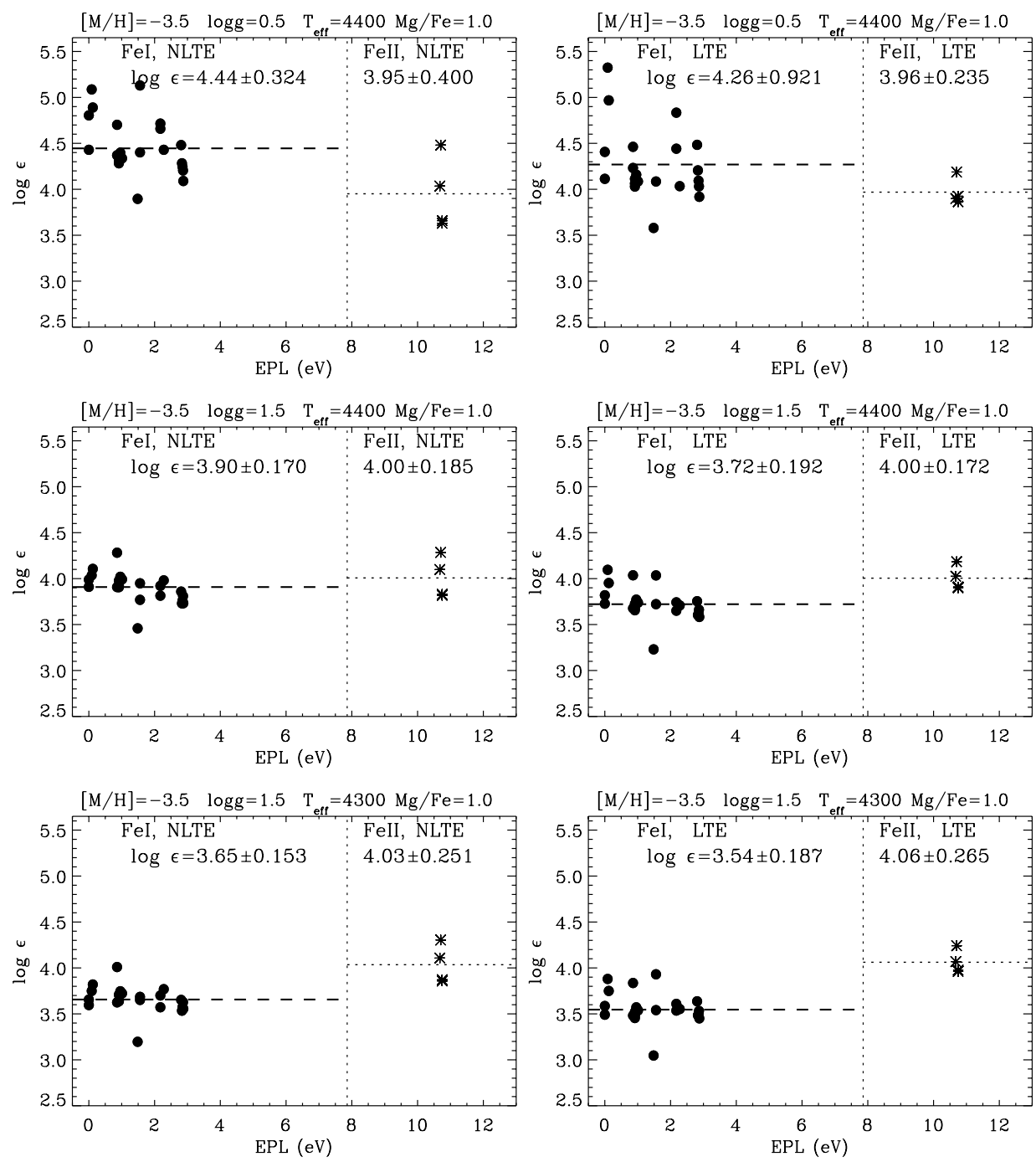

Fig. 13. Results of LTE and non-LTE Fe abundance determination for the Fe I and Fe II lines in CS 29498-043 for a small grid of model atmospheres and for $[\mathrm{Mg} / \mathrm{Fe}]=1$.

This diagram gives a broader perspective on the evolution of oxygen in the Galaxy. We propose the existence of an upper envelope of the $[\mathrm{O} / \mathrm{Fe}]$ ratio represented by the dashed line which suggests a monotonically increasing trend toward lower metallicities (Fig. 15). Very high $[\mathrm{O} / \mathrm{Fe}]$ ratios are possibly indicating that most of the Fe nuclei synthesized in the inner core are actually held by a massive compact object, i.e. a black hole. Smaller ratios possibly indicate that a significant fraction of the Fe nuclei is incorporated into the supernova ejecta, therefore a smaller mass cut is required and the likely formation of less massive compact objects, i.e. neutron stars. Assuming that below metallicity -3 , we are seeing direct yields from the first generation of supernovae, the range in $[\mathrm{O} / \mathrm{Fe}]$ encompassed by the dashed lines in our plot may be populated by stars contaminated by supernovae that led to the formation of compact objects with different masses.

The fact that the atmospheres of these stars do not contain large amounts of $\mathrm{Ca}, \mathrm{Ti}, \mathrm{Si}$ and $\mathrm{Mg}$ supports the idea of massive black holes left from the supernova explosions of the first stars. It has been suggested that the bulk of light $r$-nuclei (with $A<130$ ) appear to have different sources from those for heavy $r$-nuclei (Wasserburg et al. 1996). The meteoritic data require at least two distinct types of SN $r$-process events: the highfrequency events, $H$, producing heavy nuclei with $A>130$, including ${ }^{182} \mathrm{Hf}$, and the low-frequency $L$ events producing light nuclei with $A>130$, including ${ }^{129}$ I. The $r$-process production in the SN environments associated with the $H$ and $L$ events has been discussed in some detail by Wasserburg \& Qian (2000). The abundance analysis of CS 22949-037 and CS 29498-043 extended to heavy neutron capture elements may directly test the speculation by Wasserburg \& Qian (2000) that $H$ events are associated with supernovae producing black holes, whereas $L$ events are associated with supernovae producing neutron stars. According to this model, the parent supernovae of our targets come from the $H$-events.

The large disagreements found from different abundance indicators of $\mathrm{Mg}$ and $\mathrm{O}$ reveal that the atmospheric models used in this study are not reliable. The conflict is so severe that we 

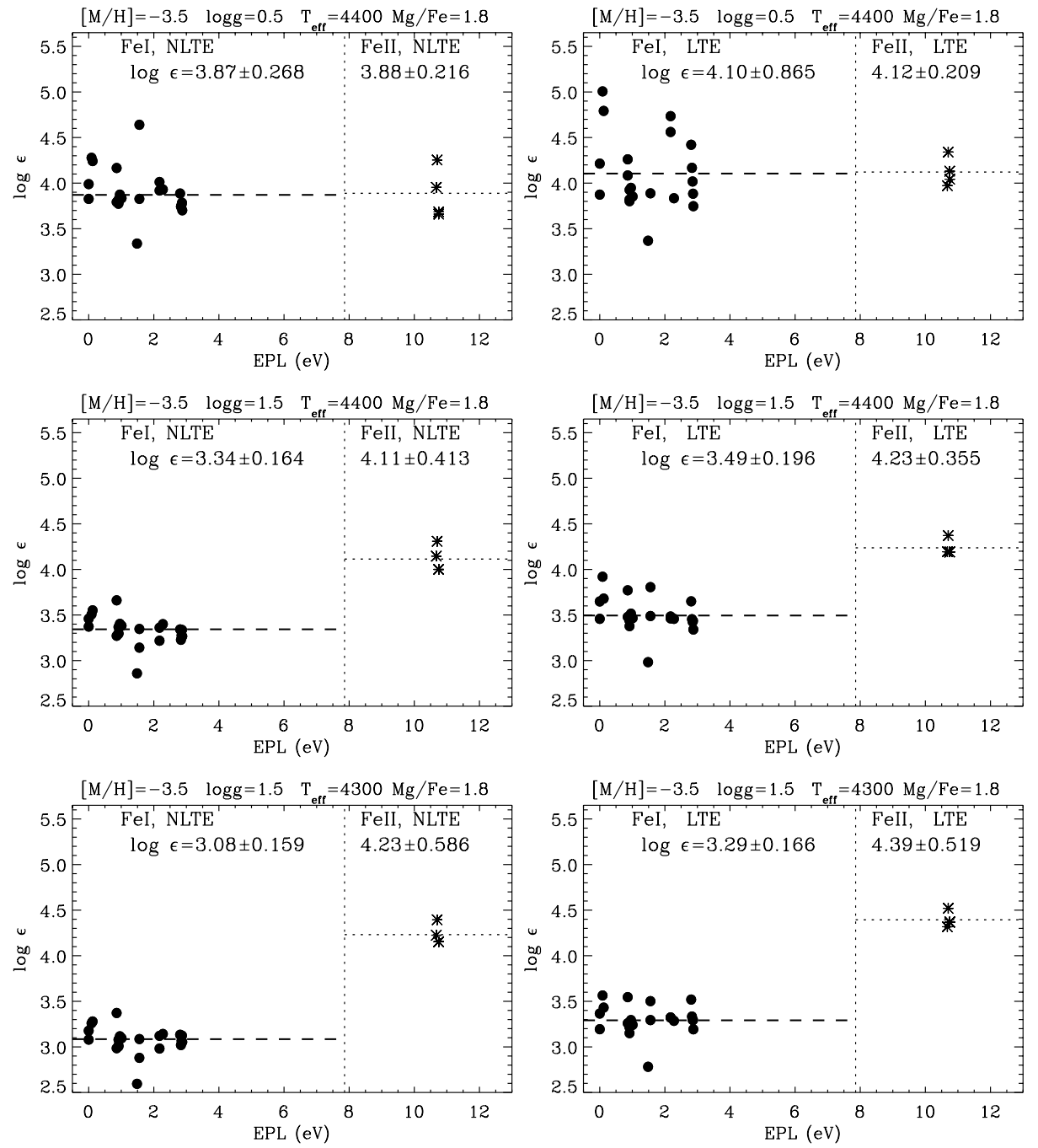

Fig. 14. The same as in Fig. 13 but for $[\mathrm{Mg} / \mathrm{Fe}]=1.8$.

cannot question the quality of the data and/or the model atoms used in our non-LTE. The problem with the oxygen and magnesium abundances may have the same roots as that discussed by Dalle Ore (1993). This author found large discrepancies among the temperatures obtained from the excitation and ionization equilibrium of several cool giants. In fact, the systematic disagreement between different temperature scales found from the continuum energy distribution, $\mathrm{H} \alpha$ and $\mathrm{Fe}$ lines is the best indication that the models employed in these studies are to some extent unreliable.

Our analysis suggests that the gravities of very metal-poor giants derived from the LTE Fe analysis are strongly underestimated because non-LTE effects are neglected. The oxygen abundances in CS 22949-037 and CS 29498-043 derived from the triplet and the forbidden line differ by a large factor. It is interesting that the oxygen forbidden line at $6300 \AA$ and the near-IR triplet are formed in the same layers deep in the atmosphere (Fig. 9). This suggests that one needs a different atmospheric structure in order to achieve consistency for $\mathrm{O}$ and Mg. It is clear that the standard 1D models of Kurucz (1992) are unreliable for ultra-metal-poor giants. As a final check of these results, we used models without convective overshooting (Castelli et al. 1997) and found that they do not resolve the discrepancy either.

\section{Conclusions}

Observations with Keck I/HIRES have revealed strong lines of the oxygen near-IR triplet in the spectra of the ultra-metalpoor giants CS 22949-037 and CS 29498-043. The forbidden line of oxygen with $E W=60 \pm 10 \mathrm{~m} \AA$ was observed with TNG/SARG. A detailed non-LTE analysis of Fe has been carried out and a new set of the atmospheric parameters have been obtained. Our analysis suggests that the gravities of metal-poor giants derived from the LTE Fe analysis are strongly underestimated because of the neglect of non-LTE effects.

The oxygen abundance in CS 22949-037 and CS29498-043 derived from the triplet and the forbidden line differ by 1.18 and 0.53 dex, respectively. This disagreement cannot be explained by a non-LTE effects, quality of the data and/or uncertainties in stellar parameters. Other mechanisms must be invoked in order to explain this puzzle. A similar discrepancy was found for $\mathrm{Mg}$ when comparing the abundances obtained from the resonance 


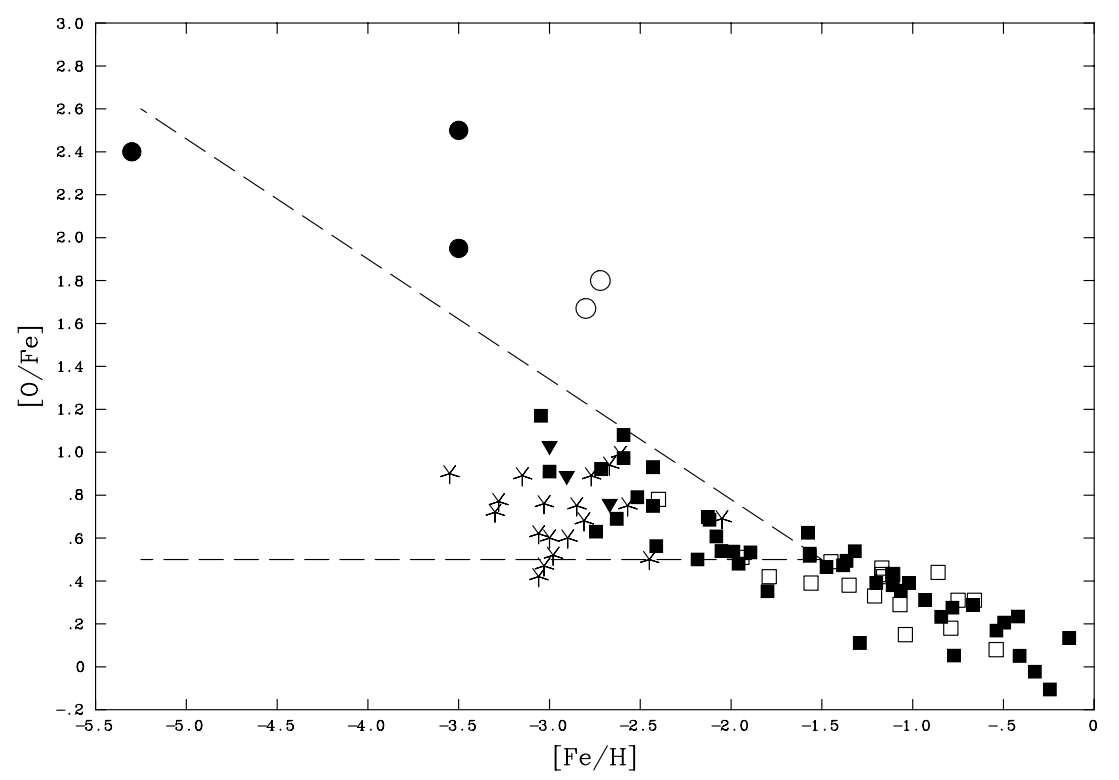

Fig. 15. Oxygen overabundance versus Fe. Data from Israelian et al. (2001), Cayrel et al. (2003), and Nissen et al. (2002) are marked by filled squares, stars, and open squares, respectively. Filled triangles represent the upper limits from Israelian et al. (2001). The values were not corrected for 3D effects because the latter do not predict consistent oxygen abundance from different indicators. We cannot incorporate these corrections in the data of Cayrel et al. (2003) since there are no 3D models available for giants. Three filled circles indicate the oxygen abundance derived from the forbidden line for the two giants discussed in this article and for HE 0107-5240 derived from UV OH lines by Bessell et al. (2004). The spectroscopic binaries CS 29497-030 $([\mathrm{Fe} / \mathrm{H}]=-2.8)$ and LP 625-44 $([\mathrm{Fe} / \mathrm{H}]=-2.72)$ with a large enhancement in $s-$ process elements are indicated by empty circles and have $[\mathrm{O} / \mathrm{Fe}]=1.67$ (Sivarani et al. 2003) and $[\mathrm{O} / \mathrm{Fe}]=1.8$ (Aoki et al. 2002b), respectively.

line $4571 \AA$ and several strong subordinate lines. Based on the present analysis we propose that the Kurucz (1992) models are not reliable for these ultra-metal-poor giants.

Acknowledgements. The data presented here were obtained with the W. M. Keck Observatory, which is operated as a scientific partnership among the California Institute of Technology, the University of California, and the National Aeronautics and Space Administration. The Observatory was made possible by the generous financial support of the W. M. Keck foundation. We are grateful to Wako Aoki and Martin Asplund for several helpful discussions and Piercarlo Bonifacio for providing interpolated models of Castelli et al. without overshooting. We thank the anonymous referee for useful suggestions and comments. N.S. would like to thank R. Kostik for several discussions and Irina Vasiljeva for a help with computations. This research was partially supported by the Spanish DGES under project AYA2001-1657 and by INTAS grant 00-00084.

\section{References}

Aoki, W., Norris, J., Ryan, S., Beers, T., \& Ando, H. 2002, PASJ, 54, 933

Aoki, W., Ando, H., Honda, S., et al. 2002b, PASJ, 54, 427

Aoki, W., Norris, J., Ryan, S., et al. 2003, ApJ, submitted

Asplund, M., Nordlund, A., Trampedach, R., \& Stein, R. F. 1999 , A\&A, 346, L17

Asplund, M., \& García Pérez, A. 2001, A\&A, 372, 601200

Beers, T., Preston, G., \& Shectman, S. A. 1992, AJ, 103, 1987

Belyaev, A. K., Grosser, J., Hahne, J., \& Menzel, T. 1999, Phys. Rev. A, 60, 2150
Bessell, M., Christlieb, N., \& Gustafsson, B. 2004, ApJ, submitted Boesgaard, A. M., King, J. R., Deliyannis, C. P., \& Vogt, S. S. 1999, AJ, 117, 492

Carlsson, M., \& Judge, P. 1993, ApJ, 402, 344

Carlsson, M., Rutten, R., \& Shcukina, N. 1992, A\&A, 253, 567

Carretta, E., Gratton, R., \& Sneden, C. 2000, A\&A, 356, 238

Carretta, E., Gratton, R., Cohen, J., Beers, T., \& Christlieb, N. 2002, AJ, 124, 481

Castelli, F., Gratton, R. G., \& Kurucz, R. L. 1997, A\&A, 318, 841

Cayrel, R, Depagne, E., Spite, M., et al. 2003, A\&A, in press

Dalle Ore, C. M. 1993, Ph.D. Thesis, University of California, Santa Cruz

Depagne, E., Spite, M., Spite, F., et al. 2002, A\&A, 390, 187

Drawin, H.-W. 1968, Z. Phys., 211, 404

Fuhr, J. R., Martin, G. A., \& Wiese, W. L. 1988, J. Phys. Chem. Ref. Data, 17, 4

Fulbright, J., \& Johnson, J. 2003, ApJ, 595, 115

Gigas, D. 1988, in The Impact of Very High S/N Spectroscopy on Stellar Physics, ed. G. Cayrel de Strobel, \& M. Spite (Dordrecht: Kluwer), Proc. of the IAU Symp., 132, 395

Gray, D. F. 1976, The observation and analysis of stellar photospheres (New York: Wiley-Interscience)

Grevesse, N., \& Sauval, A. 1998, Space Sci. Rev., 86, 161

Holweger, H., Bard, A., Kock., A., \& Kock., M. 1991, A\&A, 249, 545

Idiart, T., \& Thevenin, F. 2000, ApJ, 541, 207

Israelian, G., García López, R. J., \& Rebolo, R. 1998, ApJ, 507, 805

Israelian, G., Rebolo, R., Basri, G., Casares, J., \& Martin, E. L. 1999, Nature, 401, 142

Israelian, G., \& Rebolo, R. 2001, ApJ, 557, L43

Israelian, G., Rebolo, R., García López, R., et al. 2001, ApJ, 551, 833

Moore, Ch. E. 1959, A Multiplet Table of Astrophysical Interest, NBS Technical Note, Washington 
McWilliam, A. 1997, ARA\&A, 35, 503

McWilliam, A., Preston, G. W., Sneden, C., \& Searle, L. 1995, AJ, 109,2757

Mishenina, T., Korotin, S., Klochkova, V., \& Panchuk, V. 2000, A\&A, 353, 978

Nissen, P. E., Chen, Y. Q., Asplund, M., \& Pettini, M. 2003, A\&A, in press

Nissen, P. E., Primas, F., Asplund, M., \& Lambert, D. L. 2002, A\&A, 235,251

Norris, J. E., Ryan, S. G., \& Beers, T. C. 1997, ApJ, 488, 350

Norris, J. E., Ryan, S. G., \& Beers, T. C. 1999, ApJS, 123, 639

O’Brian, T., Wickliffe, M., Lawler, J., Whaling, W., \& Brault, J. 1991, J. Opt. Soc. Am., B8, 1185
Pastorello, A., Zampieri, L., Turatto, M., et al. 2004, MNRAS, 347, 74

Ryan, S. G., Norris, J. E., \& Beers, T. 1996, ApJ, 471, 254

Shchukina, N. 1987, Kinemat. Phys. Cel. Bod., 3(6), 36

Shchukina, N., \& Trujillo Bueno, J. 2001, ApJ, 550, 970

Sivarani, T., Bonifacio, P., Molaro, P., et al. 2004, A\&A, 413, 1073

Stephens, A., \& Boesgaard, A. 2002, AJ, 123, 1647

Takeda, Y. 2003, A\&A, 402, 343

Takada-Hidai, M., Takeda, Y., Sato, S., et al. 2002, ApJ, 573, 614

Thevenin, F., \& Idiart, T. 1999, ApJ, 521, 753

Umeda, H., \& Nomoto, K. 2003, Nature, 422, 871

Wasserburg, G. J., Busso, M., \& Gallino, R. 1996, ApJ, 466, 109

Wasserburg, G. J., \& Qian, Y.-Z. 2000, ApJ, 529, L21 